H. Umemura and H. Watanabe

Nagoya Math. J.

Vol. 148 (1997), 151-198

\title{
SOLUTIONS OF THE SECOND AND FOURTH PAINLEVÉ EQUATIONS, I
}

\author{
HIROSHI UMEMURA AND HUMIHIKO WATANABE
}

\begin{abstract}
A rigorous proof of the irreducibility of the second and fourth Painlevé equations is given by applying Umemura's theory on algebraic differential equations ([26], [27], [28]) to the two equations. The proof consists of two parts: to determine a necessary condition for the parameters of the existence of principal ideals invariant under the Hamiltonian vector field; to determine the principal invariant ideals for a parameter where the principal invariant ideals exist. Our method is released from complicated calculation, and applicable to the proof of the irreducibility of the third, fifth and sixth equation (e.g. [32]).
\end{abstract}

In previous papers [27] and [28], we settled the problem of the irreducibility of the first differential equation $P_{I}$ of Painlevé. Namely we proved that no solution of the first Painleve equation is classical. So the first Painlevé equation defines highly transcendental functions different from the classical functions. The proof depends on the condition $(\mathrm{J})$ introduced in [28], which is of arithmetic nature and plays an important role in the proof of the irreducibility of the first equation $P_{\mathrm{I}}$.

Our framework tells us that if an ordinary algebraic differential equation of second order satisfies the condition $(\mathrm{J})$, then no transcendental solution of the differential equation is classical. So for the first equation $P_{\mathrm{I}}$, the proof of the irreducibility consists of two parts: (i) To prove that the first equation satisfies the condition $(\mathrm{J})$; (ii) To show that the first Painlevé equation has no algebraic solutions.

In this paper we discuss in this framework the irreducibility of the second and fourth equations $P_{\mathrm{II}}(\alpha), P_{\mathrm{IV}}(\alpha, \beta)$ of Painlevé:

$$
\begin{aligned}
P_{\mathrm{II}}(\alpha) & \frac{d^{2} q}{d t^{2}}=2 q^{3}+t q+\alpha \\
P_{\mathrm{IV}}(\alpha, \beta) & \frac{d^{2} q}{d t^{2}}=\frac{1}{2 q}\left(\frac{d q}{d t}\right)^{2}+\frac{3}{2} q^{3}+4 t q^{2}+2\left(t^{2}-\alpha\right) q+\frac{\beta}{q} .
\end{aligned}
$$

Received May 29, 1995. 
Since, for particular values of the complex parameters $\alpha, \beta$, the equations $P_{\mathrm{II}}(\alpha)$ and $P_{\mathrm{IV}}(\alpha, \beta)$ have algebraic solutions, or classical solutions rationally expressed by solutions of Riccati equations. Our objective should be the determination of all the classical solutions of the equations. To this end, we have to do the following:

(i) To show that the equations $P_{\mathrm{II}}(\alpha), P_{\mathrm{IV}}(\alpha, \beta)$ satisfy the condition $(\mathrm{J})$ for general $\alpha, \beta$;

(ii) To determine transcendental classical solutions for particular values of the parameters $\alpha, \beta$ for which the equations $P_{\mathrm{II}}(\alpha), P_{\mathrm{IV}}(\alpha, \beta)$ do not satisfy the condition $(\mathrm{J})$;

(iii) To list up all the algebraic solutions.

We leave (iii) for a separate paper [30]. Our main results are Theorems 2.1 and 3.2. In their proofs, we use birational transformations between solutions of a Painlevé equation.

As for (iii) Murata [16] determined algebraic solutions of $P_{\mathrm{II}}(\alpha)$ and $P_{\mathrm{IV}}(\alpha, \beta)$. (i) and (ii) were done by Noumi [18] and Okamoto [21] for the second and fourth equations respectively (See also [19]). Murata [15] worked out with the third Painlevé equation. All these works were done in the above framework. But in these works the authors checked the arithmetic condition $(\mathrm{J})$ by straightforward calculations. The calculations are hard particularly in the fourth and third equations, so that there is little hope of applying their calculations to the fifth and sixth Painlevé equations. We analysed the note [21] of Okamoto on the fourth Painlevé equation (see also [19], §2) and tried to simplify his argument so that we can treat the fifth and sixth equations. We succeeded in this attempt, and we are preparing papers on solutions of the third, fifth and sixth equations (e.g. [32]). The aim of the present paper is to explain our method for the second and fourth equations.

In order to prove our main theorems, Theorems 2.1 and 3.2, it is crucial to prove Propositions 2.2 and 3.5. In the process of their proofs there are two major technical improvements. Taking Proposition 3.5, for example, we briefly explain these improvements. Let $K[p, q]$ be the polynomial ring in two variables $p$ and $q$ over an ordinary differential overfield $K$ of $\mathbf{C}(t)$. Let $X(\mathbf{v})$ be the derivation on $K[p, q]$ associated with the fourth Painlevé equation (for the definition see Subsection 3.2). The main task for proving 
the proposition is to analyse an equality

$$
X(\mathbf{v}) F=G F
$$

for some $G, F \in K[p, q]$. The first improvement is how to endow the polynomial ring $K[p, q]$ with a grading structure that simplifies the detailed analysis of (1). To this end we choose the weights of both $p$ and $q$ as one, which coincide with the order of moving poles of the solutions of the system $S_{\text {IV }}(\mathbf{v})$ of differential equations equivalent to the fourth equation. But this analytic interpretation of the weights is not necessarily satisfactory if we treat the other Painlevé equations. We show in Subsections 2.5 and 3.5 that good weights come from the Newton polygon of the Hamiltonian vector field associated with each Painlevé equation. The second improvement is the "divisibility lemmas" (Lemmas $2.3,2.4,3.6-3.8$ ). If we write $F=F_{m}+\cdots+F_{0}$ as a sum of homogeneous polynomials $F_{i}$ of weight $i$ with respect to the above grading of $K[p, q]$, then the divisibility lemmas show that the polynomials $F_{m}, F_{m-1}, F_{m-2}$, and so on, are divisible by a certain product of three monomials $p, q, p-q$. Hence we can argue as if $m=3$, so that we are released from complicated caluculation. We deduce the divisibility lemmas from several commutative diagrams ((14), (18), (22) in Subsection 3.2, etc.). These diagrams simplify the proofs of the divisibility lemmas.

The references show that there is a tradition in the study of the Painlevé equations, which deserves respect, developed in Russia and Belorus. So far as the second and fourth equations are concerned, our results look overlapped with theirs (e.g. Gromak [8], and Gromak and Tsegel'nik [10]). There are, however, two major differences: (i) We have the clear definition of classical functions; (ii) Our method is effective so that we can apply it to the fifth and sixth Painlevé equations.

\section{$\S 1$. Preliminaries}

Let $D$ be a domain of the complex line $\mathbf{C}$ with a complex coordinate $t$. We denote by $M(D)$ the field of meromorphic functions on $D . M(D)$ is an ordinary differential field with a derivation $d / d t$ and contains the field $\mathbf{C}(t)$ of rational functions of one variable $t$ as a differential subfield. In a previous paper [26] (especially Part II, §2, see also [28]) we defined a classical function. Let us review our definition.

Definition. An ordinary differential field extension $L / \mathbf{C}(t)$ is said to be a classical extension if there exist a complex domain $D$ in $\mathbf{C}$ and a finite 
sequence of elements $y_{1}, \cdots, y_{N}$ in $M(D)$ such that the following conditions are satisfied:

(i) the field $L$ is a differential subfield of the field $\mathbf{C}(t)\left\langle y_{1}, \cdots, y_{N}\right\rangle$ differentially generated over $\mathbf{C}(t)$ by the $y_{i}$ 's: $L \subseteq \mathbf{C}(t)\left\langle y_{1}, \cdots, y_{N}\right\rangle$;

(ii) for each $i(1 \leq i \leq N)$, one of the following conditions is satisfied:

(a) there are $n$ elements $a_{1}, \cdots, a_{n}$ of $\mathbf{C}(t)\left\langle y_{1}, \cdots, y_{i-1}\right\rangle$ such that $y_{i}$ is a solution of a homogeneous linear ordinary differential equation of order $n$ :

$$
\frac{d^{n} y}{d t^{n}}+a_{1} \frac{d^{n-1} y}{d t^{n-1}}+\cdots+a_{n} y=0
$$

(b) there are an Abelian function $f\left(z_{1}, \cdots, z_{n}\right)$ of $n$ variables, and $n$ elements $a_{1}, \cdots, a_{n}$ of $\mathbf{C}(t)\left\langle y_{1}, \cdots, y_{i-1}\right\rangle$ such that $y_{i}$ is the composite function $f\left(a_{1}, \cdots, a_{n}\right)$.

An element of a classical extension field is said to be a classical function.

Remark. In [26] we explained the theoretical significance of the preceding conditions (a), (b), which are related with algebraic groups. In fact we proved in [26], Part II, Theorem 2.19 that a differential field of meromorphic functions is classical if and only if it is a differential subfield of a successive $G$-primitive extension in the sense of Kolchin [12], Chap. VI.

\section{EXAMPLE.}

(i) If a function $y$ is algebraic over a classical extension field, then $y$ is classical. In particular an algebraic function is classical (cf. Lemma 1.1 in [28]).

(ii) If $f$ is a classical function, then every function $y$ satisfying

$$
\frac{d y}{d t}=f
$$

is a classical function. In fact the function $y$ satisfies a homogeneous linear ordinary differential equation

$$
f \frac{d^{2} y}{d t^{2}}-\frac{d f}{d t} \frac{d y}{d t}=0
$$

Since $f, d f / d t$ are classical, $y$ is a classical function by definition. 
(iii) For classical functions $a_{1}, a_{2}$, every function $q$ satisfying

$$
\frac{d q}{d t}+a_{1} q+a_{2}=0
$$

is a classical function. The assertion is obvious if $a_{2}=0$. Assume $a_{2} \neq 0$. A function $y(\neq 0)$ satisfying

$$
\frac{d y}{d t}+a_{1} y=0
$$

is classical by definition. If we set $q=u y$ and substitute it into (1), we have

$$
\frac{d u}{d t}=-\frac{a_{2}}{y}
$$

so that $u$ is classical by (ii). Hence $q=u y$ is a classical function.

(iv) For rational functions $a_{0}, a_{1}, a_{2} \in \mathbf{C}(t)$, every function $q$ satisfying a Riccati equation

$$
\frac{d q}{d t}+a_{0} q^{2}+a_{1} q+a_{2}=0
$$

is a classical function. If $a_{0}=0$, then $q$ is a classical function by (iii). If $a_{0} \neq 0$, then, replacing $q$ by $q / a_{0}$ if necessary, we may assume $a_{0}=1$ without loss of generality (see e.g. [11], Chap. II). If we set $q=(d / d t)(\log \tau)$, we obtain a homogeneous linear ordinary differential equation:

$$
\frac{d^{2} \tau}{d t^{2}}+a_{1} \frac{d \tau}{d t}+a_{2} \tau=0
$$

Therefore every solution of Riccati equations with coefficients in $\mathbf{C}(t)$ is a classical function.

Now we review the condition $(\mathrm{J})$. Let $K$ be an ordinary differential overfield of $\mathbf{C}(t)$ with derivation $\delta$. So the restriction of $\delta$ to $\mathbf{C}(t)$ agrees with the usual derivation $d / d t$ on $\mathbf{C}(t)$. Let $K[p, q]$ be the polynomial ring over $K$ in two variables $p$ and $q$. The derivation $\delta$ on $K$ is uniquely extended to a derivation on $K[p, q]$ such that $\delta(p)=\delta(q)=0$, which we denote by the same symbol $\delta$. For two polynomials $A(p, q), B(p, q) \in \mathbf{C}(t)[p, q] \subset K[p, q]$, we define a derivation $X$ on $K[p, q]$ by

$$
X=\delta+A(p, q) \frac{\partial}{\partial q}+B(p, q) \frac{\partial}{\partial p}: K[p, q] \longrightarrow K[p, q]
$$


So, if $K=\mathbf{C}(t)$, we have

$$
X=\frac{\partial}{\partial t}+A(p, q) \frac{\partial}{\partial q}+B(p, q) \frac{\partial}{\partial p} .
$$

We say that a polynomial $F$ in $K[p, q]$ is $X$-invariant if there exists a polynomial $G$ in $K[p, q]$ such that $X F=G F$. We also say that an ideal $I$ of $K[p, q]$ is $X$-invariant if we have $X H \in I$ for every $H \in I$. The following conditions for a principal ideal $I$ of $K[p, q]$ are equivalent:

(i) the ideal $I$ is $X$-invariant;

(ii) the ideal $I$ is generated by an $X$-invariant polynomial;

(iii) every generator of the principal ideal $I$ is $X$-invariant.

When the equivalent conditions are satisfied, the zero locus $V(I) \subset$ Spec $K[p, q]$ is called an $X$-invariant curve defined over $K$ if the principal ideal $I$ is properly between the zero-ideal and $K[p, q]: 0 \neq I \subsetneq K[p, q]$, or equivalently, if a generator $F$ of $I$ does not belong to $K$.

We introduce the following condition for $X$ :

(J) For any ordinary differential field extension $K / \mathbf{C}(t)$, there exists no $X$-invariant curve defined over $K$.

Now, let us consider the following system of ordinary differential equations:

$$
\left\{\begin{array}{l}
\frac{d q}{d t}=A(p, q), \\
\frac{d p}{d t}=B(p, q) .
\end{array}\right.
$$

We say that a solution $(p, q)$ of $(2)$ is algebraic if both $p$ and $q$ are algebraic over $\mathbf{C}(t)$. Otherwise, the solution $(p, q)$ is said to be non-algebraic or transcendental. When both $p$ and $q$ belong to $\mathbf{C}(t)$, we say that the solution $(p, q)$ is rational. We also say that a solution $(p, q)$ of $(2)$ is classical if both $p$ and $q$ are classical, and a solution $(p, q)$ is non-classical if not so. The proof of Lemma 0.8 in [27] allows us to show the following fundamental theorem that is indispensable to the classification of solutions of the Painlevé equations.

THEOREM 1.1. Let $(p, q)$ be a solution of the system (2). If the derivation $X$ satisfies the condition $(\mathrm{J})$, then one of the following conclusions holds: 
(i) the solution $(p, q)$ is algebraic;

(ii) the solution $(p, q)$ is not classical.

Namely there is no transcendental classical solution of (2).

\section{$\S 2$. The second Painlevé equation}

\subsection{Statement of the theorem}

We know that the second Painlevé equation is equivalent to the following system $S_{\mathrm{II}}(\alpha)$ of ordinary differential equations of first order:

$$
S_{\mathrm{II}}(\alpha) \quad\left\{\begin{array}{l}
\frac{d q}{d t}=p-q^{2}-\frac{t}{2} \\
\frac{d p}{d t}=2 p q+\alpha+\frac{1}{2}
\end{array}\right.
$$

where $\alpha$ is a complex parameter (cf. [20]). In fact, if we eliminate the unknown $p$ from $S_{\text {II }}(\alpha)$, we get the second Painlevé equation:

$$
P_{\mathrm{II}}(\alpha) \quad \frac{d^{2} q}{d t^{2}}=2 q^{3}+t q+\alpha .
$$

So the second Painlevé equation $P_{\mathrm{II}}(\alpha)$ and the system $S_{\mathrm{II}}(\alpha)$ are parametrized by the complex line $\mathbf{C}$.

In order to state Theorem 2.1, we review birational transformations of solutions of the system $S_{\mathrm{II}}(\alpha)$ associated with a group of complex affine transformations of the complex line $\mathbf{C}$. We define affine transformations $s$, $t_{+}, t_{-}$of $\mathbf{C}$ by $s(\alpha)=-1-\alpha, t_{-}(\alpha)=\alpha-1, t_{+}(\alpha)=\alpha+1$ for $\alpha \in \mathbf{C}$. Let $\mathbf{G}$ be the subgroup generated by them in the group of complex affine transformations of the complex line $\mathbf{C}$. Then the group $\mathbf{G}$ is isomorphic to the semidirect product of a cyclic group $\langle s\rangle$ of order two and a group $\left\langle t_{-}, t_{+}\right\rangle$. Since the latter group is isomorphic to the additive group of the integers $\mathbf{Z}$, we find $\mathbf{G} \cong \mathbf{Z} / 2 \mathbf{Z} \ltimes \mathbf{Z}$, so that it is isomorphic to the affine Weyl group of the root system of type $A_{1}$ ([1], Chap. $\left.4, \S 2,1\right)$. Let $C_{0}$ be the subset of $\mathbf{C}$ that consists of all the complex numbers $\alpha$ satisfying the following conditions:

(i) $-\frac{1}{2} \leq \Re(\alpha) \leq 0$;

(ii) $\Im(\alpha) \geq 0$ if $\Re(\alpha)=0$ or $-\frac{1}{2}$, 
where $\Re(v)$ and $\Im(v)$ denote the real and imaginary parts respectively of a complex number $v$. We see that $C_{0}$ is a fundamental region of $\mathbf{C}$ for the group $\mathbf{G}$.

For $\alpha \in \mathbf{C}$, let $\Sigma(\alpha)$ be the set of solutions $(p, q)$ of the system $S_{\mathrm{II}}(\alpha)$. Here we assume that a solution is meromorphic over a complex domain. We set $\Sigma=\bigcup_{\alpha} \Sigma(\alpha)$ (disjoint union). We define rational transformations $s_{*},\left(t_{-}\right)_{*},\left(t_{+}\right)_{*}$ of the set $\Sigma$ as follows ([20], Section 1): For $(p, q) \in \Sigma(\alpha)$,

(i) we define $s_{*}(p, q) \in \Sigma(-1-\alpha)$ by

$$
s_{*}(p, q)=\left(p, q+\frac{\alpha+\frac{1}{2}}{p}\right) \quad \text { if } \alpha \neq-\frac{1}{2},
$$

and

$$
s_{*}(p, q)=(p, q) \quad \text { if } \alpha=-\frac{1}{2}
$$

(ii) we define $\left(t_{-}\right)_{*}(p, q) \in \Sigma(\alpha-1)$ by

$$
\left(t_{-}\right)_{*}(p, q)=\left(-p+2 q^{2}+t,-q+\frac{\alpha-\frac{1}{2}}{p-2 q^{2}-t}\right) \quad \text { if } \alpha \neq \frac{1}{2},
$$

and

$$
\left(t_{-}\right)_{*}(p, q)=\left(-p+2 q^{2}+t,-q\right) \quad \text { if } \alpha=\frac{1}{2}
$$

(iii) we define $\left(t_{+}\right)_{*} \in \Sigma(\alpha+1)$ by

$$
\left(t_{+}\right)_{*}(p, q)=\left(-p+2\left(q+\frac{\alpha+\frac{1}{2}}{p}\right)^{2}+t,-q-\frac{\alpha+\frac{1}{2}}{p}\right) \quad \text { if } \alpha \neq-\frac{1}{2},
$$

and

$$
\left(t_{+}\right)_{*}(p, q)=\left(-p+2 q^{2}+t,-q\right) \quad \text { if } \alpha=-\frac{1}{2} .
$$

The definitions of $s_{*},\left(t_{-}\right)_{*},\left(t_{+}\right)_{*}$ are well-defined by the following facts: for $(p, q) \in \Sigma(\alpha)$,

(i) $p \neq 0$ if $\alpha \neq-1 / 2$;

(ii) $p-2 q^{2}-t \neq 0$ if $\alpha \neq 1 / 2$. 
In fact, the assertion (i) is trivial. If $p-2 q^{2}-t=0$, we have $0=(d / d t)(p-$ $\left.2 q^{2}-t\right)=\alpha-1 / 2$, which proves the assertion (ii).

Since we have $s_{*}^{2}=\left(t_{+}\right)_{*}\left(t_{-}\right)_{*}=\left(t_{-}\right)_{*}\left(t_{+}\right)_{*}=\mathbf{1}$, where $\mathbf{1}$ denotes the identity transformation of $\Sigma$, we see that the mappings $s_{*},\left(t_{-}\right)_{*},\left(t_{+}\right)_{*}$ define birational transformations. Let $\mathbf{G}_{*}$ be the subgroup generated by $\left(t_{+}\right)_{*}$ and $s_{*}$ in the group of all bijections of the set $\Sigma$. The group $\mathbf{G}_{*}$ consists of birational transformations of $\Sigma$ that respect the natural fibration $\pi: \Sigma \rightarrow \mathbf{C}$ defined by $\pi: \Sigma(\alpha) \ni(p, q) \rightarrow \alpha \in \mathbf{C}$. Hence we have a surjective group morphism $\varphi$ of $\mathbf{G}_{*}$ onto $\mathbf{G}$ such that $\varphi\left(s_{*}\right)=s, \varphi\left(t_{*}\right)=t$. Since $s_{*}\left(t_{+}\right)_{*} s_{*}=\left(t_{-}\right)_{*}$ and $s_{*}\left(t_{-}\right)_{*} s_{*}=\left(t_{+}\right)_{*}, \varphi$ is an isomorphism of $\mathbf{G}_{*}$ onto G. For $g \in \mathbf{G}_{*}$, the following diagram is commutative:

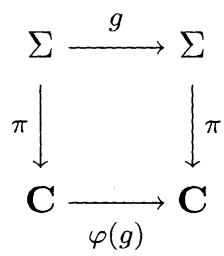

Since $g$ is $\mathbf{C}(t)$-birational, a solution $(p, q)$ is classical (resp. algebraic, rational) if and only if the solution $g(p, q)$ is classical (resp. algebraic, rational).

Now let us state our main result for the second Painlevé equation.

THEOREM 2.1. (i) For every integer $\alpha \in \mathbf{Z}$, there exists a unique rational solution of the system $S_{\mathrm{II}}(\alpha)$.

(ii) For every $\alpha \in 1 / 2+\mathbf{Z}$, there exists a unique one-parameter family of classical solutions of $S_{\mathrm{II}}(\alpha)$, of which each solution is rationally written by a solution of a Riccati equation

$$
\frac{d q}{d t}=-q^{2}-\frac{t}{2}
$$

(iii) Let $(p, q)$ be a solution of $S_{\mathrm{II}}(\alpha)$ different from those mentioned in (i) and (ii). Then neither the function $p$ nor the function $q$ is classical, and the transcendence degree of $\mathbf{C}(t, p, q)$ over $\mathbf{C}(t)$ equals two.

Using the birational transformations introduced above, we can explicitly write the solutions $(p, q)$ in the assertions (i) and (ii). In fact, if $(p, q)$ is a rational solution of $S_{\mathrm{II}}(\alpha)$ for $\alpha \in \mathbf{Z}$, then we have

$$
(p, q)=\left(t_{+}\right)_{*}^{\alpha}\left(\frac{t}{2}, 0\right) .
$$


If $(p, q)$ is one of the classical solutions of $S_{\mathrm{II}}(\alpha)(\alpha \in 1 / 2+\mathbf{Z})$ in (ii), then we have

$$
(p, q)=\left(t_{+}\right)_{*}^{\alpha+\frac{1}{2}}\left(0, q_{r}\right)
$$

where $q_{r}$ is a solution of the Riccati equation (1).

Let us introduce a new unknown $u$ by

$$
q=\frac{d}{d t}(\log u)
$$

Substituting it into (1), we obtain the Airy differential equation

$$
\frac{d^{2} u}{d t^{2}}+\frac{t}{2} u=0
$$

Hence all the classical solutions of $S_{\mathrm{II}}(\alpha)$ for $\alpha \in 1 / 2+\mathbf{Z}$ are rationally generated from Airy functions.

We explain here how we prove the theorem. Let $K$ be an ordinary differential overfield of $\mathbf{C}(t)$ with derivation $\delta$ and let $K[p, q]$ be the polynomial ring over $K$ in two variables $p$ and $q$. According to $\S 1$, we introduce a derivation $X(\alpha)$ on $K[p, q]$ by

$$
X(\alpha)=\delta+\left(p-q^{2}-\frac{t}{2}\right) \frac{\partial}{\partial q}+\left(2 p q+\alpha+\frac{1}{2}\right) \frac{\partial}{\partial p} .
$$

To prove the theorem, we may assume that the parameter $\alpha$ belongs to the fundamental domain $C_{0}$ by the operation of $\mathbf{G}$. The proof consists of the following three parts:

(I) Non-classical solutions. If there exists an $X(\alpha)$-invariant curve defined over $K\left(\alpha \in C_{0}\right)$ for any differential extention $K / \mathbf{C}(t)$, then we have $\alpha=-1 / 2$. We conclude by Theorem 1.1 that, for $\alpha \in C_{0}$ such that $\alpha \neq-1 / 2$, every solution of $S_{\mathrm{II}}(\alpha)$ is non-classical if it is not algebraic (Corollary 2.6).

(II) Classical solutions. For every $X(-1 / 2)$-invariant polynomial $F$ in $K[p, q]$ and not in $K$, there exists an integer $i>0$ such that $(F)=\left(p^{i}\right)$ (Lemma 2.7). So every transcendental classical solution of $S_{\mathrm{II}}(-1 / 2)$ is defined by the Riccati equation (1) (Proposition 2.9).

(III) Algebraic solutions. The system $S_{\mathrm{II}}(\alpha)\left(\alpha \in C_{0}\right)$ has a rational solution if and only if $\alpha=0$. The solution $(p, q)=(t / 2,0)$ is a unique rational solution of $S_{\mathrm{II}}(0)$ (In particular the assumption "if it is not algebraic" in (I) is always satisfied.). 
The procedures (I) and (II) will be done in Subsections 2.2 and 2.3. We discuss the procedure (III) in Subsection 2.4, but it will be done in a separate paper [30].

Remark. It turns out from Theorem 2.1 that the following conditions for a transcendental solution $(p, q)$ of $S_{\mathrm{II}}(\alpha)$ are equivalent:

(i) $(p, q)$ is classical;

(ii) tr.d. $[\mathbf{C}(t, p, q): \mathbf{C}(t)]=1$.

The similar result holds for the fourth Painlevé equation (cf. Theorem 3.3). Some authors determined solutions $(p, q)$ of $S_{\mathrm{II}}(\alpha)$ or $S_{\mathrm{IV}}(\mathbf{v})$ (see $\left.\S 3\right)$ with tr.d. $[\mathbf{C}(t, p, q): \mathbf{C}(t)]=1$. It is not, however, obvious at all from their determination that there is no classical solution $(p, q)$ in our sense with tr.d. $[\mathbf{C}(t, p, q): \mathbf{C}(t)]=2$. In his Stockholm Lessons [25], Painlevé had an idea of classifying transcendents by the degree of algebraic differential equation that defines the transcendents. Their determination of solutions $(p, q)$ with tr.d. $[\mathbf{C}(t, p, q) ; \mathbf{C}(t)]=1$ comes from the same idea as Painlevé's. We can not, however, measure the complexity of a function by the transcendence degree. For example a linear differential equation of order three is simpler than the Painlevé equations just as in number theory, whatever the degree may be, an abelian extension is simpler, at least theoretically, than a non-abelian extension. Generalized differential Galois Theory will give a satisfactory answer to this kind of questions (cf. [29]).

\subsection{Non-classical solutions}

The following proposition is crucial for the proof of Theorem 2.1.

Proposition 2.2. If the derivation $X(\alpha)$ does not satisfy the condition $(J)$, then there exist non-negative integers $i$ and $j$ such that

$$
i+j \geq 1
$$

and

$$
j\left(\alpha-\frac{1}{2}\right)=i\left(\alpha+\frac{1}{2}\right) .
$$

Proof. We shall proceed in six steps.

Step 1. By hypothesis there exists a differential overfield $K$ of $\mathbf{C}(t)$ such that we find an $X(\alpha)$-invariant principal ideal $I$ properly between the 
zero-ideal and $K[p, q]: 0 \neq I \subsetneq K[p, q]$. Let $F \in K[p, q]$ be a generator of $I$. So we have $I=(F), F \notin K$ and

$$
X(\alpha) F=G F
$$

for some $G \in K[p, q]$. We define the weights of $p$ and $q$ to be 2 and 1 respectively, so that the weight of a monomial $a p^{i} q^{j}(0 \neq a \in K)$ in $K[p, q]$ is $2 i+j$.

Let $R_{d}$ be the $K$-linear subspace of $K[p, q]$ generated over $K$ by all the monomials with weight $d$. Hence $K[p, q]$ becomes a graded ring: $K[p, q]=$ $\oplus_{d \geq 0} R_{d}$ with $R_{0}=K$ and $R_{d} \cdot R_{d^{\prime}} \subseteq R_{d+d^{\prime}}$. We set

$$
\begin{aligned}
X_{1} & =\left(p-q^{2}\right) \frac{\partial}{\partial q}+2 p q \frac{\partial}{\partial p} \\
X_{0} & =\delta \\
X_{-1} & =-\frac{t}{2} \frac{\partial}{\partial q} \\
X_{-2} & =\left(\alpha+\frac{1}{2}\right) \frac{\partial}{\partial p}
\end{aligned}
$$

so that $X(\alpha)=X_{1}+X_{0}+X_{-1}+X_{-2}$ is the homogeneous decomposition. Namely each $X_{i}(i=-2,-1,0,1)$ is a derivation that maps $R_{d}$ to $R_{d+i}$. Since the highest part $X_{1}$ of $X$ is of weight one and since the polynomial $F$ in (4) is not equal to zero, the polynomial $G$ in (4) belongs to the direct sum $R_{0} \oplus R_{1}$. Therefore we have $G=\mu q+\nu$ for some $\mu, \nu \in K$. Since $F \notin K$, we have an expression $F=F_{0}+\cdots+F_{m}$ with $F_{d} \in R_{d}$ and $F_{m} \neq 0$ for some integer $m \geq 1$. So the equation (4) is written as

$$
\left(X_{1}+X_{0}+X_{-1}+X_{-2}\right)\left(F_{m}+\cdots+F_{0}\right)=(\mu q+\nu)\left(F_{m}+\cdots+F_{0}\right) .
$$

Comparing the homogeneous parts of both sides of (5), we have a system of $m+4$ equations equivalent to (5), hence to (4):

$$
X_{1} F_{d}=\mu q F_{d}+\nu F_{d+1}-X_{0} F_{d+1}-X_{-1} F_{d+2}-X_{-2} F_{d+3}
$$

for each integer $d$ such that $-3 \leq d \leq m$. Here we consider $F_{-3}=F_{-2}=$ $F_{-1}=F_{m+1}=F_{m+2}=F_{m+3}=0$. In Steps 3-6, we shall recursively determine the polynomial $F_{d}$ for $d=m, m-1, m-2, m-3$. The polynomials $F_{m}, F_{m-1}, F_{m-2}$ are determined without imposing any condition on 
the parameter $\alpha$. The expected relation (3) appears as an obstruction to the determination of $F_{m-3}$.

Step 2. We need the following two auxiliary lemmas, which deliver us from a brute force calculation (cf. [18], [19], [21]).

LEMMA 2.3. Let $d$ be a non-negative integer and let $k$ be a positive integer. Let $A$ be a polynomial in $R_{d}$, and let $\mu^{\prime}$ be an element of $K$. If $\mu^{\prime}-d+4 l-4 \neq 0$ for every integer $l$ such that $1 \leq l \leq k$ and if $A$ satisfies a congruence

$$
X_{1} A \equiv \mu^{\prime} q A \quad \bmod \left(2 q^{2}-p\right)^{k},
$$

then $A \equiv 0 \bmod \left(2 q^{2}-p\right)^{k}$.

Lemma 2.4. Let $d, k, A, \mu^{\prime}$ be as above. If $\mu^{\prime}+d-4 l+4 \neq 0$ for every integer $l$ such that $1 \leq l \leq k$ and if $A$ satisfies a congruence

$$
X_{1} A \equiv \mu^{\prime} q A \quad \bmod p^{k}
$$

then $A \equiv 0 \bmod p^{k}$.

Proof of Lemma 2.3. We denote by $K[T]$ the polynomial ring in one variable $T$ over $K$. Let $\varphi$ be the $K$-algebra morphism of $K[p, q]$ onto $K[T]$ defined by $\varphi(q)=T$ and $\varphi(p)=2 T^{2}$. Then the kernel $\operatorname{Ker} \varphi$ of $\varphi$ is the principal ideal generated by $2 q^{2}-p$. The following diagram (9) is commutative:

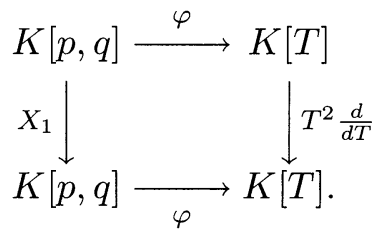

So the kernel $\operatorname{Ker} \varphi=\left(2 q^{2}-p\right)$ is $X_{1}$-invariant. In fact we have a formula

$$
X_{1}\left(2 q^{2}-p\right)=-2 q\left(2 q^{2}-p\right) \text {. }
$$

Now we show $A \equiv 0 \bmod \left(2 q^{2}-p\right)^{l}$ by induction on $l(1 \leq l \leq k)$. We set $A=\sum_{2 i+j=d} c_{i j} p^{i} q^{j}$ with $c_{i j} \in K$. Applying $\varphi$ to both sides of (7), we have

$$
\varphi\left(X_{1} A\right)=\varphi\left(\mu^{\prime} q A\right)
$$

This is equivalent to

$$
T^{2} \frac{d}{d T} \varphi(A)=\varphi\left(\mu^{\prime} q A\right)
$$


by the commutative diagram (9). Since $\varphi(A)=\left(\sum_{2 i+j=d} 2^{i} c_{i j}\right) T^{d}$, it follows that

$$
\left(\mu^{\prime}-d\right)\left(\sum_{2 i+j=d} 2^{i} c_{i j}\right) T^{d+1}=0 .
$$

Since $\mu^{\prime}-d \neq 0$ by hypothesis, we have $\sum 2^{i} c_{i j}=0$ and hence $A \equiv$ $0 \bmod 2 q^{2}-p$. This proves the case $l=1$. Assume that $A \equiv 0 \bmod \left(2 q^{2}-\right.$ $p)^{l-1}$ for $l \geq 2$. We show $A \equiv 0 \bmod \left(2 q^{2}-p\right)^{l}$. If $d<2 l-2$, we have $A=0$ since $A \in R_{d}$ is divisible by $\left(2 q^{2}-p\right)^{l-1}$, and there is nothing to prove. Therefore we may assume $d \geq 2 l-2$. Then we can put

$$
A=B\left(2 q^{2}-p\right)^{l-1}
$$

with a polynomial $B \in R_{d-2 l+2}$. If we substitute (11) into (7) and divide both sides of the resulting congruence by $\left(2 q^{2}-p\right)^{l-1}$, we get

$$
X_{1} B \equiv\left(\mu^{\prime}+2 l-2\right) q B \quad \bmod \left(2 q^{2}-p\right)^{k-l+1}
$$

If we put $B=\sum_{2 i+j=d-2 l+2} e_{i j} p^{i} q^{j}$ with $e_{i j} \in K$ and apply $\varphi$ to (12), then the same argument as above gives us a relation

$$
\left(\mu^{\prime}-d+4 l-4\right)\left(\sum_{2 i+j=d-2 l+2} 2^{i} e_{i j}\right)=0
$$

Since $\mu^{\prime}-d+4 l-4 \neq 0$, we have $\sum 2^{i} e_{i j}=0$ and hence $A \equiv 0 \bmod \left(2 q^{2}-p\right)^{l}$. This is what we had to show, and the lemma is proved.

Proof of Lemma 2.4. Let $\psi$ be the $K$-algebra morphism of $K[p, q]$ onto $K[T]$ defined by $\psi(q)=T$ and $\psi(p)=0$. The kernel Ker $\psi$ is the principal ideal generated by $p$. The following diagram (13) is commutative:



So the kernel $\operatorname{Ker} \psi=(p)$ is $X_{1}$-invariant. In fact we have a formula

$$
X_{1}(p)=2 q p
$$

The argument of the proof of Lemma 2.3 allows us to prove this lemma if we use $\psi$ and (13) for $\varphi$ and (9) respectively. Hence we omit the detail. 
Remark. The commutative diagrams (9) and (13) are obtained in the following procedure. Let us determine the homogeneous $K$-algebra morphism $\Phi$ such that the following diagram is commutative:

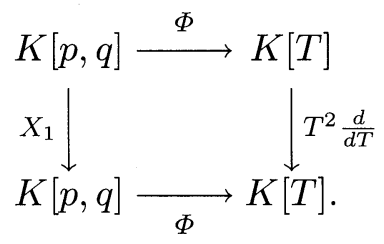

Here we consider the polynomial ring $K[T]$ as a graded ring in the usual way. Hence we can set $\Phi(p)=a T^{2}$ and $\Phi(q)=b T$ for some $a, b \in K$ and get a system of algebraic equations:

$$
\left\{\begin{aligned}
2 a b & =2 a, \\
a-b^{2} & =b .
\end{aligned}\right.
$$

Therefore we have the solutions $(a, b)=(2,1),(0,-1),(0,0)$. The first two of them define the expected morphisms $\varphi$ and $\psi$ respectively, and the remainder gives a trivial solution that has no importance.

Step 3. Now we come back to the proof of the proposition. The polynomial $F_{m}$ satisfies the equation $(6)_{m}$ :

$$
X_{1} F_{m}=\mu q F_{m}
$$

We claim that $(m-\mu) / 4$ is a nonnegative integer. In fact, otherwise, we would have $\mu-m+4 l-4 \neq 0$ for every integer $l \geq 1$. By Lemma 2.3 it would follow that $F_{m} \equiv 0 \bmod \left(2 q^{2}-p\right)^{k}$ for every integer $k \geq 1$. This contradicts the hypothesis $F_{m} \neq 0$. Moreover we claim that $(m+\mu) / 4$ is a non-negative integer. In fact, otherwise, we would have $\mu+m-4 l+4 \neq 0$ for every integer $l \geq 1$. By Lemma 2.4 it would follow that $F_{m} \equiv 0 \bmod p^{k}$ for every integer $k \geq 1$. This contradicts the hypothesis $F_{m} \neq 0$. If we put $i=(m+\mu) / 4$ and $j=(m-\mu) / 4$, we have

$$
m=2(i+j)
$$

and

$$
\mu=2(i-j)
$$

Since $m \geq 1,(15)$ implies (2) as required. If $j \geq 1$, we have $F_{m} \equiv 0 \bmod$ $\left(2 q^{2}-p\right)^{j}$ by Lemma 2.3 because $\mu-m+4 l-4 \neq 0$ for every integer $l$ such 
that $1 \leq l \leq j$. If $i \geq 1$, we have $F_{m} \equiv 0 \bmod p^{i}$ by Lemma 2.4 because $\mu+m-4 l+4 \neq 0$ for every integer $l$ such that $1 \leq l \leq i$. By (2) we have $F_{m} \equiv 0 \bmod p^{i}\left(2 q^{2}-p\right)^{j}$. Since the polynomial $p^{i}\left(2 q^{2}-p\right)^{j}$ belongs to $R_{m}$, we have $F_{m}=a p^{i}\left(2 q^{2}-p\right)^{j}$ for some non-zero element $a \in K$. The polynomial $a^{-1} F$ is $X(\alpha)$-invariant and generates the ideal $I=(F)$ introduced in Step 1. So we may assume $a=1$ and therefore

$$
F_{m}=p^{i}\left(2 q^{2}-p\right)^{j}
$$

Step 4 . The polynomial $F_{m-1}$ satisfies the equation $(6)_{m-1}$ :

$(6)_{m-1}$

$$
X_{1} F_{m-1}=\mu q F_{m-1}+\nu F_{m}-X_{0} F_{m}
$$

Since $X_{0} F_{m}=0$ by (17), the equation $(6)_{m-1}$ is rewritten as

$$
X_{1} F_{m-1}=\mu q F_{m-1}+\nu p^{i}\left(2 q^{2}-p\right)^{j},
$$

where $\mu$ is given by (16). We have $X_{1} F_{m-1} \equiv \mu q F_{m-1} \bmod p^{i}$. If $i \geq 1$, we have $F_{m-1} \equiv 0 \bmod p^{i}$ by Lemma 2.4 because $\mu+(m-1)-4 l+4=$ $4 i-4 l+3 \neq 0$ for every integer $l$ such that $1 \leq l \leq i$. Similarly we have $X_{1} F_{m-1} \equiv \mu q F_{m-1} \bmod \left(2 q^{2}-p\right)^{j}$. If $j \geq 1$, we have $F_{m-1} \equiv 0 \bmod \left(2 q^{2}-\right.$ $p)^{j}$ by Lemma 2.3 because $\mu-(m-1)+4 l-4=-4 j+4 l-3 \neq 0$ for every integer $l$ such that $1 \leq l \leq j$. By $(2)$ we have $F_{m-1} \equiv 0 \bmod p^{i}\left(2 q^{2}-p\right)^{j}$. Hence we have

$$
F_{m-1}=0
$$

It follows from (18) that we have

$$
\nu=0 \text {. }
$$

Step 5 . The polynomial $F_{m-2}$ satisfies the equation $(6)_{m-2}$ :

$(6)_{m-2} \quad X_{1} F_{m-2}=\mu q F_{m-2}+\nu F_{m-1}-X_{0} F_{m-1}-X_{-1} F_{m}$

If we substitute $(17),(19)$ and $(20)$ into $(6)_{m-2}$, we get

$$
X_{1} F_{m-2}=\mu q F_{m-2}+2 j t q p^{i}\left(2 q^{2}-p\right)^{j-1} .
$$

Since $X_{1}$ is a derivation, we have

$$
X_{1}\left(\left(2 q^{2}-p\right) F_{m-2}\right)=-2 q \cdot\left(2 q^{2}-p\right) F_{m-2}+\left(2 q^{2}-p\right) X_{1} F_{m-2}
$$


Eliminating $X_{1} F_{m-2}$ from (21) and (22), we have

(24) $X_{1}\left(\left(2 q^{2}-p\right) F_{m-2}\right)=(\mu-2) q \cdot\left(2 q^{2}-p\right) F_{m-2}+2 j t q p^{i}\left(2 q^{2}-p\right)^{j}$.

We have $X_{1}\left(\left(2 q^{2}-p\right) F_{m-2}\right) \equiv(\mu-2) q \cdot\left(2 q^{2}-p\right) F_{m-2} \bmod p^{i}$. If $i \geq 1$, we have $\left(2 q^{2}-p\right) F_{m-2} \equiv 0 \bmod p^{i}$ by Lemma 2.4 because $(\mu-2)+m-4 l+4=$ $4 i-4 l+2 \neq 0$ for every integer $l$ such that $1 \leq l \leq i$. Similarly we have $X_{1}\left(\left(2 q^{2}-p\right) F_{m-2}\right) \equiv(\mu-2) q \cdot\left(2 q^{2}-p\right) F_{m-2} \bmod \left(2 q^{2}-p\right)^{j}$. If $j \geq 1$, we have $\left(2 q^{2}-p\right) F_{m-2} \equiv 0 \bmod \left(2 q^{2}-p\right)^{j}$ by Lemma 2.3 because $(\mu-2)-m+4 l-4=-4 j+4 l-6 \neq 0$ for every integer $l$ such that $1 \leq l \leq j$. We have $\left(2 q^{2}-p\right) F_{m-2} \equiv 0 \bmod p^{i}\left(2 q^{2}-p\right)^{j}$ by $(2)$. Then there exists an element $c \in K$ such that

$$
\left(2 q^{2}-p\right) F_{m-2}=c p^{i}\left(2 q^{2}-p\right)^{j} .
$$

If we substitute (24) into (23) and notice the relation $(6)_{m}$, we have $c=j t$. We have by $(24)$

$$
F_{m-2}=j t p^{i}\left(2 q^{2}-p\right)^{j-1} .
$$

Step 6. Finally we determine the polynomial $F_{m-3}$ and derive the relation (3). $F_{m-3}$ satisfies the equation $(6)_{m-3}$ :

(6) $m-3$

$$
\begin{aligned}
X_{1} F_{m-3}= & \mu q F_{m-3}+\nu F_{m-2} \\
& -X_{0} F_{m-2}-X_{-1} F_{m-1}-X_{-2} F_{m} .
\end{aligned}
$$

If we substitute $(17),(19),(20)$ and $(25)$ into $(6)_{m-3}$, we have

$$
\begin{aligned}
X_{1} F_{m-3}= & \mu q F_{m-3} \\
& +j\left(\alpha-\frac{1}{2}\right) p^{i}\left(2 q^{2}-p\right)^{j-1}-i\left(\alpha+\frac{1}{2}\right) p^{i-1}\left(2 q^{2}-p\right)^{j} .
\end{aligned}
$$

If $m=2$, we have $i=0$ or $j=0$ by (15). Therefore we have $\alpha=1 / 2$ or $\alpha=-1 / 2$ by (26), which satisfy the relation (3). Assume $m \geq 3$. We have

$$
X_{1}\left(p\left(2 q^{2}-p\right) F_{m-3}\right)=p\left(2 q^{2}-p\right) X_{1} F_{m-3} .
$$

Substituting (26) into (27), we have

$$
\begin{aligned}
X_{1}\left(p\left(2 q^{2}-p\right) F_{m-3}\right)= & \mu q \cdot p\left(2 q^{2}-p\right) F_{m-3} \\
& +j\left(\alpha-\frac{1}{2}\right) p^{i+1}\left(2 q^{2}-p\right)^{j} \\
& -i\left(\alpha+\frac{1}{2}\right) p^{i}\left(2 q^{2}-p\right)^{j+1} .
\end{aligned}
$$


We have $X_{1}\left(p\left(2 q^{2}-p\right) F_{m-3}\right) \equiv \mu q \cdot p\left(2 q^{2}-p\right) F_{m-3} \bmod p^{i}$. If $i \geq 1$, we have $p\left(2 q^{2}-p\right) F_{m-3} \equiv 0 \bmod p^{i}$ by Lemma 2.4 because $\mu+(m+1)-$ $4 l+4=4 i-4 l+5 \neq 0$ for every integer $l$ such that $1 \leq l \leq i$. Similarly we have $X_{1}\left(p\left(2 q^{2}-p\right) F_{m-3}\right) \equiv \mu q \cdot p\left(2 q^{2}-p\right) F_{m-3} \bmod \left(2 q^{2}-p\right)^{j}$. If $j \geq 1$, we have $p\left(2 q^{2}-p\right) F_{m-3} \equiv 0 \bmod \left(2 q^{2}-p\right)^{j}$ by Lemma 2.3 because $\mu-(m+1)+4 l-4=-4 j+4 l-5 \neq 0$ for every integer $l$ such that $1 \leq l \leq j$. Therefore we have $p\left(2 q^{2}-p\right) F_{m-3} \equiv 0 \bmod p^{i}\left(2 q^{2}-p\right)^{j}$. Since $R_{1}=\{c q \mid c \in K\}$, there exists an element $c \in K$ such that

$$
p\left(2 q^{2}-p\right) F_{m-3}=c q p^{i}\left(2 q^{2}-p\right)^{j} .
$$

Substituting (29) into (28), we have

$$
\left\{c-2 i\left(\alpha+\frac{1}{2}\right)\right\} q^{2}=\left\{c-j\left(\alpha-\frac{1}{2}\right)-i\left(\alpha+\frac{1}{2}\right)\right\} p
$$

and hence we have

$$
c=2 i\left(\alpha+\frac{1}{2}\right)
$$

and

$$
i\left(\alpha+\frac{1}{2}\right)=j\left(\alpha-\frac{1}{2}\right) .
$$

Thus Proposition 2.2 is proved.

Moreover, from (29) and (30), we have

$$
\begin{aligned}
F_{m-3} & =2 i\left(\alpha+\frac{1}{2}\right) q p^{i-1}\left(2 q^{2}-p\right)^{j-1} \\
& =2 j\left(\alpha-\frac{1}{2}\right) q p^{i-1}\left(2 q^{2}-p\right)^{j-1} .
\end{aligned}
$$

Corollary 2.5. The complex number $\alpha$ in Proposition 2.2 is rational and $|\alpha| \geq 1 / 2$.

Proof. The number $\alpha$ satisfies the relation (3). We see $i \neq j$ easily. Then we have $\alpha=(i+j) /(2(i-j))$, and the assertion follows immediately.

Combining this corollary with Theorem 1.1, we deduce the next corollary immediately.

Corollary 2.6. Let $(p, q)$ be a solution of $S_{\mathrm{II}}(\alpha)$. If $\alpha \in C_{0}$ and $\alpha \neq-1 / 2$, then one of the following conclusions holds:

(i) the solution $(p, q)$ is non-classical;

(ii) the solution $(p, q)$ is algebraic. 


\subsection{Classical solutions}

We determine all the $X(-1 / 2)$-invariant principal ideals of $K[p, q]$.

LEMma 2.7. For every integer $i \geq 1$, the principal ideal $\left(p^{i}\right)$ is $X(-1 /$ $2)$-invariant. Conversely, if $I$ is an $X(-1 / 2)$-invariant principal ideal properly between the zero-ideal and $K[p, q]$, there exists an integer $i \geq 1$ such that $I=\left(p^{i}\right)$.

Proof. Let the notation be as in Proposition 2.3. The first half is obvious. For the second half, it is sufficient to show that the $X(-1 / 2)$ invariant polynomial $F$ is equal to $p^{i}$ for some integer $i \geq 1$. Since $\alpha=$ $-1 / 2$, we have $j=0$ from (3). Hence we have $m=2 i=\mu, i \geq 1, F_{m}=p^{i}$ and $F_{m-1}=F_{m-2}=F_{m-3}=0$ by (2), (15), (16), (17), (19), (25) and (31). Here we prove the next

Sublemma. Let $d$ be an integer such that $0 \leq d<2 i$ and let $A$ be $a$ polynomial in $R_{d}$. If $A$ satisfies an equation

$$
X_{1} A=2 i q A
$$

then $A=0$.

In fact, if $2 i+d \not \equiv 0 \bmod 4$, we have $2 i+d-4 l+4 \neq 0$ for every integer $l \geq 1$. Therefore we have $A=0$ by Lemma 2.4. If $2 i+d \equiv 0 \bmod 4$, then $k=(2 i+d) / 4$ is a positive integer and we have $2 i+d-4 l+4 \neq 0$ for every integer $l$ such that $1 \leq l \leq k$. It follows from Lemma 2.4 that $A \equiv 0 \bmod p^{k}$. Since $2 k-d=(2 i-d) / 2>0$, we have $A=0$, and the sublemma is proved.

Now, let $d$ be an integer such that $0 \leq d \leq m-3$, and assume $F_{d^{\prime}}=0$ for every integer $d^{\prime}$ such that $d \leq d^{\prime}<m$ (This assumption holds when $d=m-3$.). Then the polynomial $F_{d-1}$ satisfies the equation (32) for $A=F_{d-1}$. Therefore we have $F_{d-1}=0$ by Sublemma. By induction on $d$, we have $F_{d}=0$ for every integer $d$ such that $0 \leq d<m$, and the proof of the lemma is completed.

LEMMA 2.8. Let $L$ be an ordinary differential overfield of $\mathbf{C}(t)$ with derivation $\delta$, and let $K$ be an ordinary differential field with $\mathbf{C}(t) \subset K \subset L$. If a pair $(\bar{p}, \bar{q})$ of elements of $L$ satisfies the system $S_{\mathrm{II}}(-1 / 2)$, i.e.,

$$
\left\{\begin{array}{l}
\delta \bar{q}=\bar{p}-\bar{q}^{2}-, \frac{t}{2} \\
\delta \bar{p}=2 \bar{p} \bar{q}
\end{array}\right.
$$


and if the transcendence degree tr.d. $[K(\bar{p}, \bar{q}): K]$ equals one, then $\bar{p}$ equals zero, and $\bar{q}$ satisfies the Riccati equation (1):

$$
\delta \bar{q}=-\bar{q}^{2}-\frac{t}{2}
$$

Proof. The polynomial ring $K[p, q]$ is an ordinary differential ring with the derivation $X(-1 / 2)$. Let $\varphi$ be a surjective morphism of differential rings of $K[p, q]$ onto $K[\bar{p}, \bar{q}]$ sending $p$ and $q$ respectively to $\bar{p}$ and $\bar{q}$. So $\operatorname{Ker} \varphi$ is $X(-1 / 2)$-invariant. Since every prime ideal of codimension one of the polynomial ring $K[p, q]$ is principal, $\operatorname{Ker} \varphi$ is $X(-1 / 2)$-invariant principal ideal. Now it follows from Lemma $2.7 \operatorname{Ker} \varphi=(p)$, so that we have $\bar{p}=0$. Substituting $\bar{p}=0$ into (33), we find that $\bar{q}$ satisfies (1).

Let us determine all the classical solutions of the system $S_{\mathrm{II}}(-1 / 2)$.

Proposition 2.9. If a function $q(t)$ satisfies the Riccati equation (1), then $(0, q(t))$ is a classical solution of $S_{\mathrm{II}}(-1 / 2)$. Conversely, let $(p(t), q(t))$ be a transcendental classical solution of the system $S_{\mathrm{II}}(-1 / 2)$. Then $p(t)$ equals zero and $q(t)$ satisfies the Riccati equation (1).

Proof. For $\alpha=-1 / 2$, the system $S_{\mathrm{II}}(\alpha)$ is given by

$$
\left\{\begin{array}{l}
\frac{d q}{d t}=p-q^{2}-\frac{t}{2} \\
\frac{d p}{d t}=2 p q .
\end{array}\right.
$$

So the first assertion is proved. To prove the converse, we use the notation and argument of the proof of the theorem in [27], p. 787. There exists a succesive $G$-primitive extension $\mathbf{C}(t)=K_{1} \subset K_{2} \subset \cdots \subset K_{n}(n \geq 2)$ such that the field $\mathbf{C}(t, p(t), q(t))$ is a differential subfield of $K_{n}$. Since the solution $(p(t), q(t))$ is non-algebraic, there exists an index $i(2 \leq i \leq n)$ such that tr.d. $\left[K_{i}: K_{i-1}\right] \geq 1$. First, we may assume that the extension $K_{n} / K_{n-1}$ is not algebraic. Then we may also assume that either $p(t)$ or $q(t)$ is non-algebraic over $K_{n-1}$. We denote by $\bar{K}_{n-1}$ the algebraic closure of $K_{n-1}$ in $K_{n}$, so that $K_{n} / \bar{K}_{n-1}$ is a regular extension. Let $M$ be the quotient field of $K_{n} \otimes_{\bar{K}_{n-1}} K_{n}$, which is an ordinary differential field (cf. loc. cit.). We have two $\bar{K}_{n-1}$-embeddings $\varphi_{\alpha}: K_{n} \rightarrow K_{n} \otimes_{\bar{K}_{n-1}} K_{n} \subset M(\alpha=1,2)$ : $\varphi_{1}(a)=a \otimes 1, \varphi_{2}(a)=1 \otimes a$ for $a \in K_{n}$. As was shown there, we can 
find a tower of differential subfields $\varphi_{2}\left(K_{n}\right)=M_{0} \subset M_{1} \subset \cdots \subset M_{l}=M$ $(l \geq 1)$ such that tr.d. $\left[M_{i}: M_{i-1}\right] \leq 1$ for $1 \leq i \leq l$. Since either $p(t)$ or $q(t)$ is non-algebraic over $K_{n-1}$, either $\varphi_{1}(p(t))$ or $\varphi_{1}(q(t))$ is non-algebraic over $\varphi_{1}\left(K_{n-1}\right)$, and consequently either $\varphi_{1}(p(t))$ or $\varphi_{1}(q(t))$ is non-algebraic over $\varphi_{2}\left(K_{n}\right)$. Therefore there exists an index $i(1 \leq i \leq l)$ such that tr.d. $\left[M_{i-1}\left(\varphi_{1}(p(t)), \varphi_{1}(q(t))\right): M_{i-1}\right]=1$. So we see by Lemma 2.8 that $\varphi_{1}(p(t))$ equals zero, and that $\varphi_{1}(q(t))$ satisfies the Riccati equation (1), which proves the proposition in this case.

If the extension $K_{n} / K_{n-1}$ is algebraic, then we can apply the similar argument to the extension $K_{n} / K_{i}$ where $i$ denotes the integer $(1 \leq i \leq n-1)$ such that the extension $K_{n} / K_{i+1}$ is algebraic and the extension $K_{i+1} / K_{i}$ is transcendental.

\subsection{Algebraic solutions}

The algebraic solutions $q$ of the second Painlevé equation $P_{\mathrm{II}}(\alpha)$ are determined by Murata [16]. Our definition of an algebraic solution $(p, q)$ is that both the functions $p$ and $q$ are algebraic. The equation $P_{\mathrm{II}}(\alpha)$ is equivalent to the system $S_{\mathrm{II}}(\alpha)$, but there is a slight difference between these definitions. Evidently, if $(p, q)$ is an algebraic solution of $S_{\mathrm{II}}(\alpha)$, then $q$ is an algebraic solution of $P_{\mathrm{II}}(\alpha)$. But a priori there is no reason to expect the converse. In fact, as will be seen in Theorem 3.3, even if $q$ is an algebraic solution of $P_{\mathrm{IV}}(\mathbf{v}),(p, q)$ is not necessarily an algebraic solution of $S_{\mathrm{IV}}(\mathbf{v})$. For the second equation, however, we classify the algebraic solutions of $S_{\mathrm{II}}(\alpha)$, and, as a result, we know that the converse is true.

Proposition 2.10. (i) Every algebraic solution of $S_{\mathrm{II}}(\alpha)$ is rational.

(ii) The system $S_{\mathrm{II}}(\alpha)$ has a rational solution if and only if $\alpha \in 1 / 2+\mathbf{Z}$.

(iii) For each $\alpha \in 1 / 2+\mathbf{Z}$, the rational solution of $S_{\mathrm{II}}(\alpha)$ is unique.

We will prove the proposition in a forthcoming paper [30].

\subsection{The Newton polygon of the invariant polynomial $F$}

We defined a weight on $K[p, q]$ in the proof of Proposition 2.2 (Step 1). We explain where this weight comes from.

The polynomial ring $K[p, q]$ is $\mathbf{N}^{2}$-graded: If we set $R_{i j}=\left\{\gamma p^{i} q^{j} \mid \gamma \in\right.$ $K\}$ for $(i, j) \in \mathbf{N}^{2}$, then we have $K[p, q]=\bigoplus_{(i, j) \in \mathbf{N}^{2}} R_{i j}$ with $R_{k l} R_{m n}=$ $R_{k+m, l+n}$ for all $(k, l),(m, n) \in \mathbf{N}^{2}$. By the Newton polygon of a polynomial $\sum a_{k l} p^{k} q^{l} \in K[p, q]$ we understand the convex closure of the subset $\left\{(k, l) \in \mathbf{N}^{2} \mid a_{k l} \neq 0\right\}$ in the plane $\mathbf{R}^{2}$. We say that an endomorphism 
$L: K[p, q] \rightarrow K[p, q]$ of the additive group $K[p, q]$ is homogeneous of weight $(l, m)\left((l, m) \in \mathbf{Z}^{2}\right)$ if $L\left(R_{i j}\right) \subseteq R_{i+l, j+m}$ for every $(i, j) \in \mathbf{N}^{2}$. Here we regard $R_{i+l, j+m}=0$ if either $i+l$ or $j+m$ is negative. Therefore the ring $\operatorname{End}_{\mathbf{Z}} K[p, q]$ of all endomorphisms of the additive group $K[p, q]$ is a $\mathbf{Z}^{2}$-graded ring. For example derivations $p \partial / \partial q,-q^{2} \partial / \partial q, 2 p q \partial / \partial p, \delta$ are homogeneous of weight $(1,-1),(0,1),(0,1),(0,0)$, respectively. So the homogeneous decomposition of the derivation $X(\alpha)$, which is an element of $\operatorname{End}_{\mathbf{Z}} K[p, q]$, is $X(\alpha)=X_{(1,-1)}+X_{(0,1)}+X_{(0,0)}+X_{(0,-1)}+X_{(-1,0)}$, where

$$
\begin{aligned}
X_{(1,-1)} & =p \frac{\partial}{\partial q} \\
X_{(0,1)} & =-q^{2} \frac{\partial}{\partial q}+2 p q \frac{\partial}{\partial p} \\
X_{(0,0)} & =\frac{\partial}{\partial t} \\
X_{(0,-1)} & =-\frac{t}{2} \frac{\partial}{\partial q} \\
X_{(-1,0)} & =\left(\alpha+\frac{1}{2}\right) \frac{\partial}{\partial p}
\end{aligned}
$$

each $X_{(i, j)}$ being a homogeneous endomorphism of weight $(i, j)$. We define the Newton polygon of an endomorphism $L \in \operatorname{End}_{\mathbf{Z}} K[p, q]$ of the additive group $K[p, q]$ as the convex closure of the subset $\left\{(i, j) \in \mathbf{Z}^{2} \mid L_{i j} \neq\right.$ $0\}$ in $\mathbf{R}^{2}$, where the homogeneous decomposition of $L$ is given by $L=$ $\sum_{(i, j) \in \mathbf{Z}^{2}} L_{i j}$ for some $L_{i j} \in R_{i j}$. So the Newton polygon of the endomorphism $X(\alpha)$ is the convex closure of $\{(1,-1),(0,1),(0,0),(0,-1),(-1,0)\}$ in $\mathbf{R}^{2}$ :



Here $a$ (resp. $b$ ) denotes the first (resp. second) coordinate on the plane $\mathbf{R}^{2}$. We show how the Newton polygon of the endomorphism $X(\alpha) \in \operatorname{End}_{\mathbf{Z}}$ 
$(K[p, q])$ determines the Newton polygon of the $X(\alpha)$-invariant polynomial $F \in K[p, q]$.

Since the $\mathbf{N}^{2}$-grading is not easy to treat, we introduced in the proof of Proposition 2.2 an $\mathrm{N}$-grading on $K[p, q]$ to analyze the equation (4). To understand the significance of this grading, we define a family of grading structures on $K[p, q]$ : We define the weights of $p$ and $q$ to be $\rho$ and $\sigma$ respectively, which are integers such that $\sigma>0$ and $-\sigma<\rho<2 \sigma$. By definition the weight of a monomial $a p^{i} q^{j}(0 \neq a \in K)$ in $K[p, q]$ is $i \rho+j \sigma$. Let $R_{d}^{\prime}$ be the $K$-linear subspace of $K[p, q]$ generated by all the monomials of weight $d$. Hence $K[p, q]$ becomes a graded ring with respect to this weight: $K[p, q]=\bigoplus_{d} R_{d}^{\prime}$ with $R_{d}^{\prime} \cdot R_{d^{\prime}}^{\prime} \subseteq R_{d+d^{\prime}}^{\prime}$. If we decompose the polynomial $F$ with respect to this gradation of $K[p, q]$, we have the homogeneous decomposition

$$
F=F_{n^{\prime}}^{\prime}+\cdots+F_{n}^{\prime}
$$

with $F_{d}^{\prime} \in R_{d}^{\prime}$ and $F_{n}^{\prime} \neq 0$ for some integers $n^{\prime}, n\left(n^{\prime} \leq n\right)$. If we set

$$
\begin{aligned}
X_{\sigma}^{\prime} & =-q^{2} \frac{\partial}{\partial q}+2 p q \frac{\partial}{\partial p} \\
X_{\rho-\sigma}^{\prime} & =p \frac{\partial}{\partial q} \\
X_{0}^{\prime} & =\frac{\partial}{\partial t} \\
X_{-\rho}^{\prime} & =\left(\alpha+\frac{1}{2}\right) \frac{\partial}{\partial p} \\
X_{-\sigma}^{\prime} & =-\frac{t}{2} \frac{\partial}{\partial q}
\end{aligned}
$$

then we see that $X(\alpha)=X_{\sigma}^{\prime}+X_{\rho-\sigma}^{\prime}+X_{0}^{\prime}+X_{-\rho}^{\prime}+X_{-\sigma}^{\prime}$ and each $X_{i}^{\prime}(i=$ $\sigma, \rho-\sigma, 0,-\rho,-\sigma)$ is a derivation mapping $R_{d}^{\prime}$ to $R_{d+i}^{\prime}$. The homogeneous decomposition of the endomorphism $X(\alpha): K[p, q] \rightarrow K[p, q]$ with respect to this grading is independent of $\rho, \sigma$. Then the equation (4) is written as

$$
\begin{aligned}
\left(X_{\sigma}^{\prime}+X_{\rho-\sigma}^{\prime}+X_{0}^{\prime}+X_{-\rho}^{\prime}\right. & \left.+X_{-\sigma}^{\prime}\right)\left(F_{n^{\prime}}^{\prime}+\cdots+F_{n}^{\prime}\right) \\
& =(\mu q+\nu)\left(F_{n^{\prime}}^{\prime}+\cdots+F_{n}^{\prime}\right) .
\end{aligned}
$$

We notice here $\sigma>\max \{\rho-\sigma, 0\}$. Comparing the homogeneous parts of the maximum weight $\sigma+n$ of both sides of (35), we have an equation for $F_{n}^{\prime}$ :

$$
X_{\sigma}^{\prime} F_{n}^{\prime}=\mu q F_{n}^{\prime}
$$


where $\mu$ is given by (16). We set $F_{n}^{\prime}=\sum_{k \rho+l \sigma=n} c_{k l} p^{k} q^{l}$ with $c_{k l} \in K$. Substituting this into $(36)$, we have the relations $(2 k-l-\mu) c_{k l}=0$ for integers $k, l \in \mathbf{N}$ such that $k \rho+l \sigma=n$. Since $F_{n}^{\prime} \neq 0$, there exist nonnegative integers $k_{0}$ and $l_{0}$ such that $c_{k_{0} l_{0}} \neq 0$. So we have

$$
\mu=2 k_{0}-l_{0}
$$

If $c_{k^{\prime} l^{\prime}} \neq 0$ for other $k^{\prime}$ and $l^{\prime}$, we have a simultaneous equation:

$$
\left\{\begin{array}{l}
\mu=2 k_{0}-l_{0}=2 k^{\prime}-l^{\prime}, \\
n=\rho k_{0}+\sigma l_{0}=\rho k^{\prime}+\sigma l^{\prime} .
\end{array}\right.
$$

Since the determinant $\left|\begin{array}{cc}2 & -1 \\ \rho & \sigma\end{array}\right|=2 \sigma+\rho \neq 0$, we have $k_{0}=k^{\prime}$ and $l_{0}=l^{\prime}$, and therefore the polynomial $F_{n}^{\prime}$ is given by

$$
F_{n}^{\prime}=c_{k_{0} l_{0}} p^{k_{0}} q^{l_{0}}
$$

This shows that $\left(k_{0}, l_{0}\right)$ is a vertex of the Newton polygon of $F$, denoted by $P$,and that the Newton polygon is contained in a subset

$$
\left\{(a, b) \in \mathbf{R}^{2} \mid b-l_{0} \leq-2\left(a-k_{0}\right), a-k_{0} \leq-\left(b-l_{0}\right)\right\}
$$

as in the figure below:

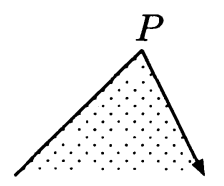

In the Newton polygon of $X(\alpha)$ in Figure 1, as in Figure 2, there is no edge with a vertex $B$ and of slope $\tau$ such that $-2<\tau<1$. Now we take the weight introduced in the proof of Proposition 2.2 that comes from the edge $B C$ of the Newton polygon of the derivation $X(\alpha)$ (see Figure 1). Then the highest component of the derivation $X(\alpha)$ is $\left(p-q^{2}\right)(\partial / \partial q)+2 p q(\partial / \partial p)$, and we found the polynomial $F_{m}$ as the highest component of the invariant polynomial $F$ (see $(17)$ ). In Figure 2 , the polynomial $F_{m}$ gives an edge with the vertex $P$ lying on a line

$$
\left\{(a, b) \in \mathbf{R}^{2} \mid b-l_{0}=-2\left(a-k_{0}\right)\right\}
$$


Therefore two monomials, $F_{n}^{\prime}$ and $2^{j} p^{i} q^{2 j}$ in $F_{m}$, represent the same vertex $P$, and hence are equal. Thus we have the relations

$$
\begin{aligned}
k_{0} & =i, \\
l_{0} & =2 j, \\
c_{k_{0} l_{0}} & =2^{j} .
\end{aligned}
$$

If we repeat this procedure at each vertex of the Newton polygon of the derivation $X(\alpha)$, we can determine the whole figure of the Newton polygon of the invariant polynomial $F$, which is the intersection of the first quadrant and a quadrilateral $P Q R S$ such that the slope of the edges $P Q, Q R, R S$, $S P$ are respectively $1,-1,0,-2$ as is shown below:



Here $a$ (resp. $b$ ) denotes the first (resp. second) coordinate on $\mathbf{R}^{2}$.

Remark. The Newton polygon of an invariant polynomial can be degenerate. In fact, we have an $X(-1 / 2)$-invariant polynomial $p$ whose Newton polygon is a subset $\{(1,0)\} \subseteq \mathbf{R}^{2}$. Moreover, we have an $X(1 / 2)$ invariant polynomial $2 q^{2}-p+t$ whose Newton polygon is represented by the following picture:

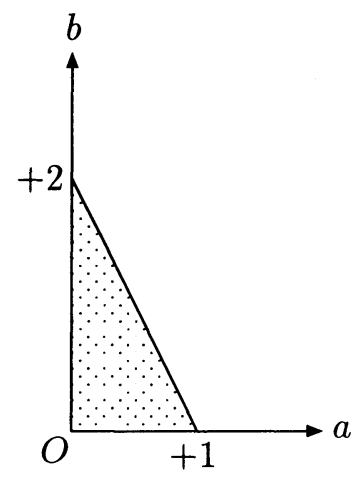


These Newton polygons are degenerate forms of the Newton polygon in Figure 3.

\section{$\S 3$. The fourth Painlevé equation}

\subsection{Statement of the theorem}

Now we treat the following system $S_{\mathrm{IV}}(\mathbf{v})$ of ordinary differential equations:

$$
S_{\mathrm{IV}}(\mathbf{v}) \quad\left\{\begin{array}{l}
\frac{d q}{d t}=2 p q-q^{2}-2 t q+2\left(v_{1}-v_{2}\right), \\
\frac{d p}{d t}=2 p q-p^{2}+2 t p+2\left(v_{1}-v_{3}\right),
\end{array}\right.
$$

where $\mathbf{v}=\left(v_{1}, v_{2}, v_{3}\right)$ denotes an arbitrary vector on a complex plane $V$ in $\mathbf{C}^{3}$ defined by $v_{1}+v_{2}+v_{3}=0([20])$. The system $S_{\mathrm{IV}}(\mathbf{v})$ is equivalent to the fourth Painlevé equation:

$$
P_{\mathrm{IV}}(\alpha, \beta) \quad \frac{d^{2} q}{d t^{2}}=\frac{1}{2 q}\left(\frac{d q}{d t}\right)^{2}+\frac{3}{2} q^{3}+4 t q^{2}+2\left(t^{2}-\alpha\right) q+\frac{\beta}{q} .
$$

In fact if we eliminate the unknown $p$ from $S_{\mathrm{IV}}(\mathbf{v})$, we get $P_{\mathrm{IV}}(\alpha, \beta)$ under the relations: $\alpha=3 v_{3}+1, \beta=-2\left(v_{2}-v_{1}\right)^{2}$. In order to state Theorem 3.2, we review birational transformations of solutions of the system $S_{\text {IV }}(\mathbf{v})$ associated with a group of affine transformations of the complex plane $V$. We define three affine transformations $s_{1}, s_{2}, t_{-}$of $V$ by $s_{1}(\mathbf{v})=\left(v_{2}, v_{1}, v_{3}\right), s_{2}(\mathbf{v})=\left(v_{3}, v_{2}, v_{1}\right), t_{-}(\mathbf{v})=\mathbf{v}+(1 / 3)(-1,-1,2)$ for $\mathbf{v}=\left(v_{1}, v_{2}, v_{3}\right) \in V$. We have $s_{1}^{2}=s_{2}^{2}=\mathbf{1}, t_{-} s_{1}=s_{1} t_{-}$, where 1 denotes the identity transformation of $V$. If we set $s_{0}=t_{-}^{-1} s_{1} s_{2} s_{1} t_{-}, z_{0}=s_{1} s_{2} t_{-}$, we have $s_{0}(\mathbf{v})=\left(v_{1}, v_{3}+1, v_{2}-1\right)$ and $z_{0}(\mathbf{v})=\left(v_{2}-1 / 3, v_{3}+2 / 3, v_{1}-1 / 3\right.$. We also have $s_{0}^{2}=z_{0}^{3}=\mathbf{1}, t_{-}^{-1} s_{2} t_{-}=s_{1} s_{0} s_{1}$. Let $\mathbf{G}$ be the subgroup generated by $s_{1}, s_{2}, t_{-}$in the group of all complex affine transformations of $V$. We can also choose $s_{1}, s_{2}, z_{0}$ as generators of the group $\mathbf{G}$. Let $\mathbf{H}$ be the subgroup of $\mathbf{G}$ generated by $s_{0}, s_{1}, s_{2}$. Setting $V^{\prime}=V \cap \mathbf{R}^{3}$, we have $V_{\mathbf{C}}^{\prime}=V^{\prime} \otimes_{\mathbf{R}} \mathbf{C}=V$. The group $\mathbf{H}$ of affine transformations of $V$ leaves the real form $V^{\prime}$ invariant. The operation of $\mathbf{H}$ on the real space $V^{\prime}$ is isomorphic to that of the affine Weyl group of the root system of type $A_{2}$ on the two dimensional real vector space (cf. [1], Chap. VI.). We easily see that $\mathbf{H}$ is a normal subgroup of $\mathbf{G}$, and we have a group isomorphism $\mathbf{G} \cong \mathbf{H} \rtimes\left\langle z_{0}\right\rangle$. Let $\Gamma$ be the subset of $V$ that consists of all the vectors $\mathbf{v}=\left(v_{1}, v_{2}, v_{3}\right)$ satisfying the following conditions: 
(i) $\Re\left(v_{2}-v_{1}\right) \geq 0$;

(ii) $\Re\left(v_{1}-v_{3}\right) \geq 0$;

(iii) $\Re\left(v_{3}-v_{2}+1\right) \geq 0$;

(iv) $\Im\left(v_{2}-v_{1}\right) \geq 0$ if $\Re\left(v_{2}-v_{1}\right)=0$;

(v) $\Im\left(v_{1}-v_{3}\right) \geq 0$ if $\Re\left(v_{1}-v_{3}\right)=0$;

(vi) $\Im\left(v_{3}-v_{2}\right) \geq 0$ if $\Re\left(v_{3}-v_{2}+1\right)=0$.

Here $\Re(v)$ and $\Im(v)$ denote the real and imaginary parts respectively of a complex number $v$.

LEMMA 3.1. The subset $\Gamma$ is a fundamental region of $V$ for the group H.

Proof. We set $V^{\prime}=V \cap \mathbf{R}^{3}$ and $\Gamma^{\prime}=\Gamma \cap \mathbf{R}^{3}$. The subset $\Gamma^{\prime}$ is a fundamental region of the real vector space $V^{\prime}$ for the group $\mathbf{H}$, because the set $\Gamma^{\prime}$ is the closure of an alcove of the affine Weyl group $\mathbf{H}$ (cf. [1], Chap. VI, $\S 2)$. We set $\tilde{\Gamma}=\left\{\mathbf{v} \in V \mid \Re\left(v_{2}-v_{1}\right) \geq 0, \Re\left(v_{1}-v_{3}\right) \geq 0\right.$, and $\Re\left(v_{3}-v_{2}+1\right) \geq$ $0\}$. We have $\tilde{\Gamma} \supset \Gamma$, and the interior of $\tilde{\Gamma}$ agrees with that of $\Gamma$. Every $\mathbf{H}$ orbit on $V$ contains a point of $\tilde{\Gamma}$, because $\Gamma^{\prime}$ is a fundamental region of $V^{\prime}$. We show that the intersection of each $\mathbf{H}$-orbit $\Omega$ and the subset $\Gamma$ consists of one point. It is easy to see that this fact proves the lemma. The difference between $\Gamma$ and $\tilde{\Gamma}$ consists of boundary. So we may assume that the $\mathbf{H}$-orbit $\Omega$ contains a point of boundary. For example, let us analyse what happens on a boundary stratum

$$
B_{3}=\left\{\mathbf{v} \in \tilde{\Gamma} \mid \Re\left(v_{2}-v_{1}\right)=0, \Re\left(v_{1}-v_{3}\right) \Re\left(v_{3}-v_{2}+1\right) \neq 0\right\} .
$$

The stratum $B_{3}$ is $s_{1}$-invariant. If we set

$$
\begin{aligned}
& B_{3}^{+}=\left\{\mathbf{v} \in B_{3} \mid \Im\left(v_{2}-v_{1}\right)>0\right\} \\
& B_{3}^{0}=\left\{\mathbf{v} \in B_{3} \mid \Im\left(v_{2}-v_{1}\right)=0\right\} \\
& B_{3}^{-}=\left\{\mathbf{v} \in B_{3} \mid \Im\left(v_{2}-v_{1}\right)<0\right\}
\end{aligned}
$$

then we have a decomposition $B_{3}=B_{3}^{+} \cup B_{3}^{0} \cup B_{3}^{-}$(disjoint union). The restriction of $s_{1}$ to $B_{3}^{0}$ is the identity mapping on $B_{3}^{0}$, and we have $s_{1}\left(B_{3}^{+}\right)=$ 
$B_{3}^{-}$and $s_{1}\left(B_{3}^{-}\right)=B_{3}^{+}$. So, if $\Omega \cap B_{3} \neq \varnothing$, then $\Omega \cap\left(B_{3}^{+} \cup B_{3}^{0}\right) \neq \varnothing$, so that $\Omega \cap \Gamma \neq \varnothing$. Similarly a boundary stratum

$$
\begin{aligned}
& B_{23}=\left\{\mathbf{v} \in \tilde{\Gamma} \mid \Re\left(v_{2}-v_{1}\right)=\Re\left(v_{1}-v_{3}\right)=0,\right. \\
& \text { or, equivalently, } \left.\Re\left(v_{1}\right)=\Re\left(v_{2}\right)=\Re\left(v_{3}\right)=0\right\}
\end{aligned}
$$

is $\mathbf{W}$-invariant, where $\mathbf{W}$ denotes the subgroup of $\mathbf{H}$ generated by $s_{1}, s_{2}$. We have

$$
B_{23}=\bigcup_{g \in \mathbf{W}} g\left(\left\{\mathbf{v} \in \Gamma \mid \Re\left(v_{1}\right)=\Re\left(v_{2}\right)=\Re\left(v_{3}\right)=0\right\}\right) .
$$

Let us prove this equality. The set $B_{23}$ is regarded as a two-dimensional real vector space of all vectors $\mathbf{u}=\left(u_{1}, u_{2}, u_{3}\right)$ such that $u_{1}+u_{2}+u_{3}=0$. The group $\mathbf{W}$ effectively acts on it as a subgroup of affine transformations, and is isomorphic to the Weyl group of the root system of type $A_{2}$. Since the set $\left\{\mathbf{v} \in \Gamma \mid \Re\left(v_{1}\right)=\Re\left(v_{2}\right)=\Re\left(v_{3}\right)=0\right\}$ is regarded as the closure of a Weyl chamber with respect to this action, we easily have the equality above. Now, if $\Omega \cap B_{23} \neq \varnothing$, then $\Omega \cap\left\{\mathbf{v} \in \Gamma \mid \Re\left(v_{1}\right)=\Re\left(v_{2}\right)=\Re\left(v_{3}\right)=0\right\} \neq \varnothing$ by the equality above, so that $\Omega \cap \Gamma \neq \varnothing$. Applying the above argument to every boundary stratum of $\tilde{\Gamma}$, we have $\Omega \cap \Gamma \neq \varnothing$.

To complete the proof we have to show that for every $\mathbf{H}$-orbit $\Omega$ the set $\Omega \cap \Gamma$ consists of one point. Let us show $\mathbf{v}_{1}=\mathbf{v}_{2}$ for $\mathbf{v}_{1}, \mathbf{v}_{2} \in \Omega \cap \Gamma$. There exists $g \in \mathbf{H}$ such that $\mathbf{v}_{1}=g\left(\mathbf{v}_{2}\right)$. In particular we have $\Re\left(\mathbf{v}_{1}\right)=$ $g \Re\left(\mathbf{v}_{2}\right)$, where $\Re(\mathbf{v})$ denotes the real vector $\left(\Re\left(v_{1}\right), \Re\left(v_{2}\right), \Re\left(v_{3}\right)\right)$ in $V^{\prime}$ for $\mathbf{v}=\left(v_{1}, v_{2}, v_{3}\right) \in V$. If $\Re\left(\mathbf{v}_{1}\right)$ is in the interior of $\Gamma^{\prime}$, then the equality $\Re\left(\mathbf{v}_{1}\right)=g \Re\left(\mathbf{v}_{2}\right)$ implies $g=\mathbf{1}$, so that $\mathbf{v}_{1}=\mathbf{v}_{2}$. Assume that $\Re\left(\mathbf{v}_{1}\right)$ lies on the boundary of $\Gamma^{\prime}$, for example, $\Re\left(\mathbf{v}_{1}\right) \in B_{3} \cap \Gamma^{\prime}$. Then we have $g=\mathbf{1}$ or $s_{1}$. Therefore we have $\mathbf{v}_{1}, \mathbf{v}_{2} \in B_{3}$ with either $\mathbf{v}_{1}=s_{1}\left(\mathbf{v}_{2}\right)$ or $\mathbf{v}_{1}=\mathbf{v}_{2}$. We may assume $\mathbf{v}_{1}=s_{1}\left(\mathbf{v}_{2}\right)$. Then it follows from the decomposition $B_{3}=B_{3}^{+} \cup B_{3}^{0} \cup B_{3}^{-}$that $\mathbf{v}_{1}, \mathbf{v}_{2} \in B_{3}^{0}$, so that $\mathbf{v}_{1}=s_{1}\left(\mathbf{v}_{2}\right)=\mathbf{v}_{2}$. Thus Lemma 3.1 is proved.

Now, let $C_{0}$ be the subset of $V$ that consists of all the vectors $\mathbf{v}=$ $\left(v_{1}, v_{2}, v_{3}\right)$ satisfying the following conditions:

(i) $\Re\left(v_{1}-v_{3}\right) \geq 0$;

(ii) $\Re\left(v_{2}+v_{3}\right) \geq 0$;

(iii) $\Re\left(v_{3}+1 / 3\right) \geq 0$; 
(iv) $\Im\left(v_{1}-v_{3}\right) \geq 0$ if $\Re\left(v_{1}-v_{3}\right)=0$;

(v) $\Im\left(v_{2}+v_{3}\right) \geq 0$ if $\Re\left(v_{2}+v_{3}\right)=0$;

(vi) $\Im\left(v_{3}\right) \geq 0$ if $\Re\left(v_{3}+1 / 3\right)=0$.

Since $\Gamma=C_{0} \cup z_{0} C_{0} \cup z_{0}^{2} C_{0}$ and $\mathbf{G} \cong \mathbf{H} \rtimes\left\langle z_{0}\right\rangle$, we have the following properties by Lemma 3.1:

(i) $V=\bigcup_{g \in \mathbf{G}} g\left(C_{0}\right)$;

(ii) for $g, h \in \mathbf{G}(g \neq h)$ the interior of $g\left(C_{0}\right)$ does not intersect that of $h\left(C_{0}\right)$.

We define two subsets $W$ and $D$ of $V$ by

$$
\begin{aligned}
W= & \left\{\mathbf{v} \in V \mid v_{1}-v_{2} \in \mathbf{Z}\right\} \\
& \cup\left\{\mathbf{v} \in V \mid v_{2}-v_{3} \in \mathbf{Z}\right\} \cup\left\{\mathbf{v} \in V \mid v_{3}-v_{1} \in \mathbf{Z}\right\}, \\
D= & \left\{\mathbf{v} \in V \mid v_{1}-v_{2} \in \mathbf{Z}\right\} \\
& \cap\left\{\mathbf{v} \in V \mid v_{2}-v_{3} \in \mathbf{Z}\right\} \cap\left\{\mathbf{v} \in V \mid v_{3}-v_{1} \in \mathbf{Z}\right\} .
\end{aligned}
$$

They are G-invariant subsets of $V$. A subset $C_{0} \cap W=C_{0} \cap\left\{\mathbf{v} \in V \mid v_{1}=\right.$ $\left.v_{3}\right\}$ is a fundamental region of $W$ for $\mathbf{G}$. The set $D$ is an orbit of the origin $\mathbf{0}$ of $V$ by the group $\mathbf{G}: D=\mathbf{G} \cdot \mathbf{0}$.

For $\mathbf{v} \in V$, let $\Sigma(\mathbf{v})$ be the set of solutions $(p, q)$ of $S_{\mathrm{IV}}(\mathbf{v})$. We set $\Sigma=\cup_{\mathbf{v}} \Sigma(\mathbf{v})$ (disjoint union). We define three birational transformations $\left(s_{1}\right)_{*},\left(s_{2}\right)_{*},\left(t_{-}\right)_{*}$ of the set $\Sigma$ as follows (cf. [20]): For $(p, q) \in \Sigma(\mathbf{v})$, (i) we define $\left(s_{1}\right)_{*}(p, q) \in \Sigma\left(s_{1}(\mathbf{v})\right)$ by

$$
\left(s_{1}\right)_{*}(p, q)=\left(p+\frac{2\left(v_{1}-v_{2}\right)}{q}, q\right) \quad \text { if } v_{1}-v_{2} \neq 0
$$

and

$$
\left(s_{1}\right)_{*}(p, q)=(p, q) \quad \text { if } v_{1}-v_{2}=0
$$

(ii) we define $\left(s_{2}\right)_{*}(p, q) \in \Sigma\left(s_{2}(\mathbf{v})\right)$ by

$$
\left(s_{2}\right)_{*}(p, q)=\left(p, q+\frac{2\left(v_{1}-v_{3}\right)}{p}\right) \quad \text { if } v_{1}-v_{3} \neq 0
$$

and

$$
\left(s_{2}\right)_{*}(p, q)=(p, q) \quad \text { if } v_{1}-v_{3}=0
$$


(iii) we define $\left(t_{-}\right)_{*}(p, q) \in \Sigma\left(t_{-}(\mathbf{v})\right)$ by

$$
\begin{aligned}
\left(t_{-}\right)_{*}(p, q)= & \left(-\frac{q(p-q-2 t)+2\left(v_{3}-v_{2}+1\right)}{p-q-2 t}\right. \\
& \left.(p-q-2 t) \frac{q(p-q-2 t)+2\left(v_{1}-v_{2}\right)}{q(p-q-2 t)+2\left(v_{3}-v_{2}+1\right)}\right)
\end{aligned}
$$

if $\left(v_{1}-v_{3}-1\right)\left(v_{2}-v_{3}-1\right) \neq 0$,

$$
\left(t_{-}\right)_{*}(p, q)=\left(-q, p-q-2 t+\frac{2\left(v_{1}-v_{2}\right)}{q}\right)
$$

if $v_{2}-v_{3}-1=0$ and $v_{1}-v_{3}-1 \neq 0$,

$$
\left(t_{-}\right)_{*}(p, q)=\left(-q-\frac{2\left(v_{1}-v_{2}\right)}{p-q-2 t}, p-q-2 t\right)
$$

if $v_{1}-v_{3}-1=0$ and $v_{2}-v_{3}-1 \neq 0$, and

$$
\left(t_{-}\right)_{*}(p, q)=(-q, p-q-2 t)
$$

if $v_{1}-v_{3}-1=v_{2}-v_{3}-1=0$ (i.e. $\mathbf{v}=(1 / 3,1 / 3,-2 / 3)$ ).

The preceding definitions of $\left(s_{1}\right)_{*},\left(s_{2}\right)_{*},\left(t_{-}\right)_{*}$ are well-defined by the following facts: for each $(p, q) \in \Sigma(\mathbf{v})$,

(i) $q \neq 0$ if $v_{1}-v_{2} \neq 0$;

(ii) $p \neq 0$ if $v_{1}-v_{3} \neq 0$;

(iii) $p-q-2 t \neq 0$ if $v_{2}-v_{3}-1 \neq 0$;

(iv) $q(p-q-2 t)+2\left(v_{3}-v_{2}+1\right) \neq 0$ if $\left(v_{2}-v_{3}-1\right)\left(v_{1}-v_{3}-1\right) \neq 0$.

The assertions are proved in the same way as in the case of the second Painlevé equation (cf. Subsection 2.1). We omit the detail.

Let $\mathbf{G}_{*}$ be the subgroup generated by $\left(s_{1}\right)_{*},\left(s_{2}\right)_{*},\left(t_{-}\right)_{*}$ in the group of all bijections of the set $\Sigma$. The group $\mathbf{G}_{*}$ consists of birational transformations of $\Sigma$. There exists a surjective group morphism $f$ of $\mathbf{G}_{*}$ onto $\mathbf{G}$ such that $f\left(\left(s_{1}\right)_{*}\right)=s_{1}, f\left(\left(s_{2}\right)_{*}\right)=s_{2}, f\left(\left(t_{-}\right)_{*}\right)=t_{-}$. We set $\mathbf{H}_{*}=f^{-1}(\mathbf{H})$. Let $\pi$ be the natural projection of $\Sigma$ onto $V$ defined by $\pi(p, q)=\mathbf{v}$ for $(p, q) \in \Sigma(\mathbf{v})$. Then the following diagram is commutative for every $g \in \mathbf{G}_{*}$ :

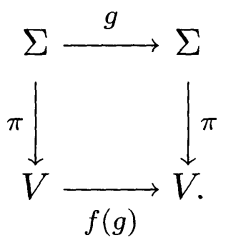


Let us now state our principal theorem for the fourth Painlevé equation.

TheOREM 3.2. (i) For every vector $\mathbf{v}$ in $W$ and not in $D$, there exists a one-parameter family of classical solutions of the system $S_{\mathrm{IV}}(\mathbf{v})$.

(ii) For every $\mathbf{v} \in D$, there exist two one-parameter families of classical solutions of the system $S_{\mathrm{IV}}(\mathbf{v})$.

(iii) Let $(p, q)$ be a transcendental solution of $S_{\mathrm{IV}}(\mathbf{v})(\mathbf{v} \in V)$ different from those in (i) and (ii). Then neither the function $p$ nor the function $q$ is classical, and the transcendence degree of $\mathbf{C}(t, p, q)$ over $\mathbf{C}(t)$ equals two.

As we shall see in Theorem 3.3 below, we have tr.d. $[\mathbf{C}(t, p, q): \mathbf{C}(t)] \leq 1$ for every solution $(p, q)$ in (i), (ii).

To prove Theorem 3.2, we may assume the vector $\mathbf{v}$ parametrizing the system $S_{\mathrm{IV}}(\mathbf{v})$ belongs to the fundamental region $\Gamma$ of the group $\mathbf{H}$ by the operation of the group $\mathbf{H}_{*}$ on the set of solutions. Consequently, it is sufficient to prove the following theorem, in which we explicitly determine all the transcendental classical solutions of $S_{\mathrm{IV}}(\mathbf{v})$ for every $\mathbf{v} \in \Gamma$ for which $S_{\mathrm{IV}}(\mathbf{v})$ has such solutions.

THEOREM 3.3. (i) For every $\mathbf{v}_{1}=\left(v_{1}, v_{2}, v_{3}\right) \in V$ such that $v_{1}=v_{3}$ and for every solution $q$ of a Riccati equation

$$
\frac{d q}{d t}=-q^{2}-2 t q+2\left(v_{1}-v_{2}\right)
$$

$(0, q)$ is a classical solution of $S_{\mathrm{IV}}\left(\mathbf{v}_{1}\right)$.

(ii) For every $\mathbf{v}_{2}=\left(v_{1}, v_{2}, v_{3}\right) \in V$ such that $v_{1}=v_{2}$ and for every solution p of a Riccati equation

$$
\frac{d p}{d t}=-p^{2}+2 t p+2\left(v_{1}-v_{3}\right)
$$

$(p, 0)$ is a classical solution of $S_{\mathrm{IV}}\left(\mathbf{v}_{2}\right)$.

(iii) For every $\mathbf{v}_{3}=\left(v_{1}, v_{2}, v_{3}\right) \in V$ such that $v_{2}=v_{3}+1$ and for every solution $q$ of a Riccati equation

$$
\frac{d q}{d t}=q^{2}+2 t q+2\left(v_{1}-v_{2}\right)
$$

$(q+2 t, q)$ is a classical solution of $S_{\mathrm{IV}}\left(\mathbf{v}_{3}\right)$.

(iv) Let $(p, q)$ be a transcendental solution of $S_{\mathrm{IV}}(\mathbf{v})(\mathbf{v} \in \Gamma)$ different from those in (i)-(iii). Then neither the function $p$ nor the function $q$ is classical, and the transcendence degree of $\mathbf{C}(t, p, q)$ over $\mathbf{C}(t)$ equals two. 
The assertions (i)-(iii) are obvious. The assertion (iv) is proved in Subsection 3.4.

Using the birational transformations in the group $\mathbf{H}_{*}$, we can explicitly write every classical solution in Theorem 3.2 as a rational function of one of the classical solutions in (i)-(iii) of Theorem 3.3. In fact, let $(p, q)$ be a classical solution of $S_{\mathrm{IV}}(\mathbf{v})$ for a $\mathbf{v} \in W$. Since $\Gamma \cap W$ is a fundamental region of an $\mathbf{H}$-invariant subset $W$ of $V$, there exist an element $g \in \mathbf{H}$ and a unique vector $\mathbf{v}_{0} \in \Gamma \cap W$ such that $\mathbf{v}=g\left(\mathbf{v}_{0}\right)$. Therefore, there exists a classical solution $\left(p_{0}, q_{0}\right)$ of $S_{\mathrm{IV}}\left(\mathbf{v}_{0}\right)$ in Theorem 3.3 and an element $\tilde{g} \in f^{-1}(g)$ such that $(p, q)=\tilde{g}\left(p_{0}, q_{0}\right)$.

Moreover, we notice the following fact.

LEMMA 3.4. The three Riccati equations (1)-(3) are birationally equivalent each other through the birational transformation $\left(z_{0}\right)_{*}$.

Proof. Let the notation be as in Theorem 3.3. The proof is divided into the following three parts.

(i) Let $(0, q)$ be a classical solution of $S_{\mathrm{IV}}\left(\mathbf{v}_{1}\right)$ defined by (1). Then a solution $\left(z_{0}\right)_{*}(0, q)=(-q,-q-2 t)$ belongs to $\Sigma\left(z_{0}\left(\mathbf{v}_{1}\right)\right)$, where the vector $z_{0}\left(\mathbf{v}_{1}\right)=\left(v_{2}-1 / 3, v_{3}+2 / 3, v_{1}-1 / 3\right)$ is in $\Gamma \cap\left\{\mathbf{v} \in V \mid v_{2}=v_{3}+1\right\}$. If we set $Q=-q-2 t$, we see that $Q$ satisfies a Riccati equation

$$
\frac{d Q}{d t}=Q^{2}+2 t Q+2\left(v_{2}-v_{3}-1\right)
$$

which is equal to (3) with $\mathbf{v}_{3}=z_{0}\left(\mathbf{v}_{1}\right)$.

(ii) Let $(p, 0)$ be a classical solution of $S_{\mathrm{IV}}\left(\mathbf{v}_{2}\right)$ defined by (2). Then a solution $\left(z_{0}\right)_{*}(p, 0)=(0, p-2 t)$ belongs to $\Sigma\left(z_{0}\left(\mathbf{v}_{2}\right)\right)$, where the vector $z_{0}\left(\mathbf{v}_{2}\right)=\left(v_{2}-1 / 3, v_{3}+2 / 3, v_{1}-1 / 3\right)$ is in $\Gamma \cap\left\{\mathbf{v} \in V \mid v_{1}=v_{3}\right\}$. If we set $Q=p-2 t$, we see that $Q$ satisfies a Riccati equation

$$
\frac{d Q}{d t}=-Q^{2}-2 t Q+2\left(v_{2}-v_{3}-1\right)
$$

which is equal to (1) with $\mathbf{v}_{1}=z_{0}\left(\mathbf{v}_{2}\right)$.

(iii) Let $(q+2 t, q)$ be a classical solution of $S_{\mathrm{IV}}\left(\mathbf{v}_{3}\right)$ defined by (3). Then a solution $\left(z_{0}\right)_{*}(q+2 t, q)=(-q, 0)$ belongs to $\Sigma\left(z_{0}\left(\mathbf{v}_{3}\right)\right)$, where the vector $z_{0}\left(\mathbf{v}_{3}\right)=\left(v_{2}-1 / 3, v_{3}+2 / 3, v_{1}-1 / 3\right)$ is in $\Gamma \cap\left\{\mathbf{v} \in V \mid v_{1}=v_{2}\right\}$. If we set $P=-q$, we see that $P$ satisfies a Riccati equation

$$
\frac{d P}{d t}=-P^{2}+2 t P+2\left(v_{2}-v_{1}\right)
$$

which is equal to (2) with $\mathbf{v}_{2}=z_{0}\left(\mathbf{v}_{3}\right)$. 
Let us introduce a new unknown $u$ by

$$
q=\frac{d}{d t}(\log u)
$$

If we eliminate the unknown $q$ from (1) and (4), we have the Hermite differential equation for $u$

$$
\frac{d^{2} u}{d t^{2}}+2 t \frac{d u}{d t}+2\left(v_{1}-v_{2}\right) u=0
$$

Therefore, we see by Lemma 3.4 that all the solutions of the Riccati equations (1)-(3), and therefore all the classical solutions of $S_{\mathrm{IV}}(\mathbf{v})$ for each $\mathbf{v} \in W$, are rationally generated from Hermite functions defined by (5).

\subsection{Non-classical solutions}

Let $K$ be an ordinary differential overfield of $\mathbf{C}(t)$, and let $K[p, q]$ be the polynomial ring over $K$ in two variables $p$ and $q$. We consider the following derivation $X(\mathbf{v})$ on $K[p, q]$ :

$$
\begin{aligned}
X(\mathbf{v})= & \frac{\partial}{\partial t}+\left(2 p q-q^{2}-2 t q+2 v_{1}-2 v_{2}\right) \frac{\partial}{\partial q} \\
& +\left(2 p q-p^{2}+2 t p+2 v_{1}-2 v_{3}\right) \frac{\partial}{\partial p} .
\end{aligned}
$$

Proposition 3.5. If there exists a vector $\mathbf{v}=\left(v_{1}, v_{2}, v_{3}\right) \in V$ for which $X(\mathbf{v})$ does not satisfy the condition $(\mathrm{J})$, then there exist nonnegative integers $i, j, k$ such that

$$
i+j+k \geq 1
$$

and

$$
i\left(v_{1}-v_{3}\right)+j\left(v_{2}-v_{1}\right)+k\left(1+v_{3}-v_{2}\right)=0 .
$$

Proof. We shall proceed in five steps.

Step 1. By hypothesis there exists a differential overfield $K$ of $\mathbf{C}(t)$ such that there exists an $X(\mathbf{v})$-invariant principal ideal $I$ properly between the zero-ideal and $K[p, q]$. Let $F \in K[p, q]$ be a generator of $I$. So we have $I=(F), F \notin K$ and

$$
X(\mathbf{v}) F=G F
$$

for some $G \in K[p, q]$. We define both the weights of $p$ and $q$ to be one, so that the weight of a monomial $a p^{i} q^{j}(0 \neq a \in K)$ in $K[p, q]$ is $i+j$. Let $R_{d}$ 
be the $K$-linear subspace of $K[p, q]$ generated over $K$ by all the monomials of weight $d$. Hence $K[p, q]$ becomes a graded ring: $K[p, q]=\bigoplus_{d \geq 0} R_{d}$ with $R_{0}=K$ and $R_{d} \cdot R_{d^{\prime}} \subseteq R_{d+d^{\prime}}$. We set

$$
\begin{aligned}
X_{1} & =(2 q-p) p \frac{\partial}{\partial p}+(2 p-q) q \frac{\partial}{\partial q} \\
X_{0} & =\frac{\partial}{\partial t}+2 t p \frac{\partial}{\partial p}-2 t q \frac{\partial}{\partial q} \\
X_{-1} & =2\left(v_{1}-v_{3}\right) \frac{\partial}{\partial p}+2\left(v_{1}-v_{2}\right) \frac{\partial}{\partial q}
\end{aligned}
$$

so that $X(\mathbf{v})=X_{1}+X_{0}+X_{-1}$ is the homogeneous decomposition. Namely each $X_{i}(i=-1,0,1)$ is a derivation that maps $R_{d}$ to $R_{d+i}$. Since the highest part $X_{1}$ of $X(\mathbf{v})$ is of weight one and since the polynomial $F$ in (8) is not equal to zero, the polynomial $G$ in (8) belongs to a direct sum $R_{0} \oplus R_{1}$. Therefore we have $G=\lambda p+\mu q+g$ for some $\lambda, \mu, g \in K$. Since $F \notin K$, we have an expression: $F=F_{0}+\cdots+F_{m}$ with $F_{d} \in R_{d}$ and $F_{m} \neq 0$ for some integer $m \geq 1$. So the equation (8) is written as

(9) $\left(X_{1}+X_{0}+X_{-1}\right)\left(F_{0}+\cdots+F_{m}\right)=(\lambda p+\mu q+g)\left(F_{0}+\cdots+F_{m}\right)$.

Comparing the homogeneous parts of both sides of (9), we have a system of $m+3$ equations equivalent to (9):

$$
X_{1} F_{d}=(\lambda p+\mu q) F_{d}+g F_{d+1}-X_{0} F_{d+1}-X_{-1} F_{d+2}
$$

for each integer $d$ such that $-2 \leq d \leq m$. Here we consider $F_{-2}=F_{-1}=$ $F_{m+1}=F_{m+2}=0$.

Step 2. Before treating the equations $(10)_{d}$, we shall show the following three auxiliary lemmas.

LEMMA 3.6. Let $d$ be a non-negative integer and let $k$ be a positive integer. Let $A$ be a polynomial in $R_{d}$, and let $\lambda^{\prime}$ and $\mu^{\prime}$ be elements of $K$. If $\lambda^{\prime}+\mu^{\prime}-d+3 l-3 \neq 0$ for every integer $l$ such that $1 \leq l \leq k$ and if $A$ satisfies a congruence

$$
X_{1} A \equiv\left(\lambda^{\prime} p+\mu^{\prime} q\right) A \quad \bmod (p-q)^{k}
$$

then $A \equiv 0 \bmod (p-q)^{k}$. 
LEMma 3.7. Let $d, k, A, \lambda^{\prime}, \mu^{\prime}$ be as above. If $\mu^{\prime}+d-3 l+3 \neq 0$ for every integer $l$ such that $1 \leq l \leq k$ and if $A$ satisfies a congruence

$$
X_{1} A \equiv\left(\lambda^{\prime} p+\mu^{\prime} q\right) A \bmod p^{k},
$$

then $A \equiv 0 \bmod p^{k}$.

Lemma 3.8. Let $d, k, A, \lambda^{\prime}, \mu^{\prime}$ be as above. If $\lambda^{\prime}+d-3 l+3 \neq 0$ for every integer $l$ such that $1 \leq l \leq k$ and if $A$ satisfies a congruence

$$
X_{1} A \equiv\left(\lambda^{\prime} p+\mu^{\prime} q\right) A \bmod q^{k} .
$$

then $A \equiv 0 \bmod q^{k}$.

Proof of Lemma 3.6. We denote by $K[T]$ the polynomial ring in one variable $T$ over $K$. Let $\varphi$ be the $K$-algebra morphism of $K[p, q]$ onto $K[T]$ defined by $\varphi(p)=\varphi(q)=T$. The kernel $\operatorname{Ker} \varphi$ of $\varphi$ is the principal ideal generated by $p-q$. The following diagram (14) is commutative:

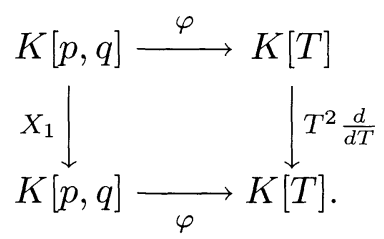

So the kernel $\operatorname{Ker} \varphi=(p-q)$ is $X_{1}$-invariant. In fact we have a formula

$$
X_{1}(p-q)=-(p+q)(p-q) .
$$

Now we show $A \equiv 0 \bmod (p-q)^{l}$ by induction on $l(1 \leq l \leq k)$. We set $A=\sum_{i+j=d} c_{i j} p^{i} q^{j}$ with $c_{i j} \in K$. Applying $\varphi$ to both sides of (11), we have

$$
\varphi\left(X_{1} A\right)=\varphi\left(\left(\lambda^{\prime} p+\mu^{\prime} q\right) A\right)
$$

This is equivalent to

$$
T^{2} \frac{d}{d T} \varphi(A)=\varphi\left(\lambda^{\prime} p+\mu^{\prime} q\right) \varphi(A)
$$

by the commutative diagram (14). Since $\varphi(A)=\left(\sum_{i+j=d} c_{i j}\right) T^{d}$, it follows that

$$
\left(\lambda^{\prime}+\mu^{\prime}-d\right)\left(\sum_{i+j=d} c_{i j}\right) T^{d+1}=0
$$


Since $\lambda^{\prime}+\mu^{\prime}-d \neq 0$ by hypothesis, we have $\sum c_{i j}=0$ and hence $A \equiv$ $0 \bmod p-q$. This proves the case $l=1$. Assume that $A \equiv 0 \bmod (p-q)^{l-1}$ for $l \geq 2$. We show $A \equiv 0 \bmod (p-q)^{l}$. If $d<l-1$, we have $A=0$ because $A \in R_{d}$ is divisible by $(p-q)^{l-1}$, and there is nothing to prove. Therefore we may assume $d \geq l-1$. Then we can put

$$
A=B(p-q)^{l-1}
$$

with a polynomial $B \in R_{d-l+1}$. If we substitute (16) into (11) and divide both sides of the resulting congruence by $(p-q)^{l-1}$, we get

$$
X_{1} B \equiv\left\{\left(\lambda^{\prime}+l-1\right) p+\left(\mu^{\prime}+l-1\right) q\right\} B \quad \bmod (p-q)^{k-l+1} .
$$

If we put $B=\sum_{i+j=d-l+1} e_{i j} p^{i} q^{j}$ with $e_{i j} \in K$ and apply $\varphi$ to (17), then the same argument as above gives us a relation

$$
\left(\lambda^{\prime}+\mu^{\prime}-d+3 l-3\right)\left(\sum_{i+j=d-l+1} e_{i j}\right)=0 .
$$

Since $\lambda^{\prime}+\mu^{\prime}-d+3 l-3 \neq 0$, we have $\sum e_{i j}=0$ and hence $A \equiv 0 \bmod (p-q)^{l}$. Thus Lemma 3.6 is proved.

Proof of Lemma 3.7. Let $\psi_{p}$ be the $K$-algebra morphism of $K[p, q]$ onto $K[T]$ defined by $\psi_{p}(p)=0$ and $\psi_{p}(q)=T$. The kernel Ker $\psi_{p}$ is the principal ideal generated by $p$. The following diagram (18) is commutative:



So the kernel Ker $\psi_{p}=(p)$ is $X_{1}$-invariant. In fact we have a formula

$$
X_{1}(p)=(2 q-p) p
$$

Now we show $A \equiv 0 \bmod p^{l}$ by induction on $l(1 \leq l \leq k)$. We set $A=\sum_{i+j=d} c_{i j} p^{i} q^{j}$ with $c_{i j} \in K$. Applying $\psi_{p}$ to both sides of (12), we have

$$
\psi_{p}\left(X_{1} A\right)=\psi_{p}\left(\mu^{\prime} q A\right)
$$


This is equivalent to

$$
-T^{2} \frac{d}{d T} \psi_{p}(A)=\psi_{p}\left(\mu^{\prime} q A\right)
$$

by the diagram (18). Since $\psi_{p}(A)=c_{0 d} T^{d}$, it follows that

$$
\left(\mu^{\prime}+d\right) c_{0 d} T^{d+1}=0 .
$$

Since $\mu^{\prime}+d \neq 0$ by hypothesis, we have $c_{0 d}=0$ and hence $A \equiv 0 \bmod p$. This proves the case $l=1$. Assume that $A \equiv 0 \bmod p^{l-1}$ for $l \geq 2$. We show $A \equiv 0 \bmod p^{l}$. If $d<l-1$, we have $A=0$ because $A \in R_{d}$ is divisible by $p^{l-1}$, and there is nothing to prove. Therefore we may assume $d \geq l-1$. Then we can put

$$
A=B p^{l-1}
$$

with a polynomial $B \in R_{d-l+1}$. If we substitute (20) into (12) and divide both sides of the resulting congruence by $p^{l-1}$, then we get

$$
X_{1} B \equiv\left\{\left(\lambda^{\prime}+l-1\right) p+\left(\mu^{\prime}-2 l+2\right) q\right\} B \quad \bmod p^{k-l+1} .
$$

If we put $B=\sum_{i+j=d-l+1} e_{i j} p^{i} q^{j}$ with $e_{i j} \in K$ and apply $\psi_{p}$ to $(21)$, then the same argument as above gives us a relation:

$$
\left(\mu^{\prime}+d-3 l+3\right) e_{0, d-l+1}=0 .
$$

Since $\mu^{\prime}+d-3 l+3 \neq 0$, we have $e_{0, d-l+1}=0$ and hence $A \equiv 0 \bmod p^{l}$. Thus the lemma is proved.

Proof of Lemma 3.8. Let $\psi_{q}$ be the $K$-algebra morphism of $K[p, q]$ onto $K[T]$ defined by $\psi_{q}(q)=0$ and $\psi_{q}(p)=T$. The kernel Ker $\psi_{q}$ is the principal ideal generated by $q$. Then the following diagram (22) is commutative:

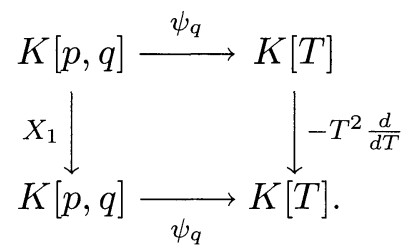

So the kernel $\operatorname{Ker} \psi_{q}=(q)$ is $X_{1}$-invariant. In fact we have a formula

$$
X_{1}(q)=(2 p-q) q .
$$

The argument of the proof of Lemma 3.7 allows us to prove this lemma if we use $\psi_{q}$ and (22) for $\psi_{p}$ and (18) respectively. Hence we omit the detail. 
Remark. The commutative diagrams (14), (18), (22) are obtained in the following procedure. Let us determine the homogeneous $K$-algebra morphism $\Phi$ such that the following diagram is commutative:



Here we consider the polynomial ring $K[T]$ as a graded ring in the usual way. Hence we can set $\Phi(p)=a T$ and $\Phi(q)=b T$ for some $a, b \in K$, and get a system of algebraic equations:

$$
\left\{\begin{array}{c}
(2 b-a) a=a, \\
(2 a-b) b=b .
\end{array}\right.
$$

Therefore we have the solutions $(a, b)=(1,1),(0,-1),(-1,0),(0,0)$. The first three of them define the expected morphisms $\phi, \psi_{p}, \psi_{q}$ respectively, and the remainder gives a trivial solution that has no importance.

Step 3. Let us come back to the proof of the proposition. The polynomial $F_{m}$ satisfies the equation $(10)_{m}$ :

$$
X_{1} F_{m}=(\lambda p+\mu q) F_{m}
$$

We claim that $(m-\lambda-\mu) / 3$ is a non-negative integer. In fact, otherwise, we would have $\lambda+\mu-m+3 l-3 \neq 0$ for every integer $l \geq 1$. By Lemma 3.6, it would follow that $F_{m} \equiv 0 \bmod (p-q)^{k}$ for every integer $k \geq 1$. This contradicts the hypothesis $F_{m} \neq 0$. Similarly we see that $(\mu+m) / 3$ and $(\lambda+m) / 3$ are non-negative integers by Lemmas 3.7 and 3.8 respectively. If we put $i=(\mu+m) / 3, j=(\lambda+m) / 3$ and $k=(m-\lambda-\mu) / 3$, then we have

$$
\begin{aligned}
& i+j+k=m, \\
& \lambda=3 j-m, \\
& \mu=3 i-m .
\end{aligned}
$$

Since $m \geq 1,(24)$ implies (6) as required. If $i \geq 1$, we have $F_{m} \equiv 0 \bmod p^{i}$ by Lemma 3.7 because $\mu+m-3 l+3 \neq 0$ for every integer $l$ such that $1 \leq l \leq i$. If $j \geq 1$, we have $F_{m} \equiv 0 \bmod q^{j}$ by Lemma 3.8 because 
$\lambda+m-3 l+3 \neq 0$ for every integer $l$ such that $1 \leq l \leq j$. If $k \geq 1$, we have $F_{m} \equiv 0 \bmod (p-q)^{k}$ by Lemma 3.6 because $\lambda+\mu-m+3 l-3 \neq 0$ for every integer $l$ such that $1 \leq l \leq k$. By (6) we have $F_{m} \equiv 0 \bmod p^{i} q^{j}(p-q)^{k}$. Since the polynomial $p^{i} q^{j}(p-q)^{k}$ belongs to $R_{m}$, we have $F_{m}=a p^{i} q^{j}(p-q)^{k}$ for some non-zero element $a \in K$. The polynomial $a^{-1} F$ is $X(\mathbf{v})$-invariant and generates the ideal $I=(F)$ introduced in Step 1. So we may assume $a=1$ and therefore

$$
F_{m}=p^{i} q^{j}(p-q)^{k} .
$$

Step 4 . The polynomial $F_{m-1}$ satisfies the equation $(10)_{m-1}$ :

$(10)_{m-1}$

$$
X_{1} F_{m-1}=(\lambda p+\mu q) F_{m-1}+g F_{m}-X_{0} F_{m} .
$$

If we substitute $(27)$ into $(10)_{m-1}$, we get

$$
\begin{aligned}
X_{1} F_{m-1}= & (\lambda p+\mu q) F_{m-1}+\{g-2(i-j) t\} p^{i} q^{j}(p-q)^{k} \\
& -2 k t(p+q) p^{i} q^{j}(p-q)^{k-1},
\end{aligned}
$$

where $\lambda$ and $\mu$ are given by (25) and (26). Since $X_{1}$ is a derivation, we have

$$
X_{1}\left((p-q) F_{m-1}\right)=-(p+q)(p-q) F_{m-1}+(p-q) X_{1} F_{m-1} .
$$

Eliminating $X_{1} F_{m-1}$ from (28) and (29), we have

$$
\begin{aligned}
X_{1}\left((p-q) F_{m-1}\right)=\{ & (\lambda-1) p+(\mu-1) q\}(p-q) F_{m-1} \\
& +\{g-2(i-j) t\} p^{i} q^{j}(p-q)^{k+1} \\
& -2 k t(p+q) p^{i} q^{j}(p-q)^{k} .
\end{aligned}
$$

We have $X_{1}\left((p-q) F_{m-1}\right) \equiv\{(\lambda-1) p+(\mu-1) q\}(p-q) F_{m-1} \bmod p^{i}$. If $i \geq 1$, we have $(p-q) F_{m-1} \equiv 0 \bmod p^{i}$ by Lemma 3.7 because $(\mu-1)+m-3 l+3=$ $3 i-3 l+2 \neq 0$ for every integer $l$ such that $1 \leq l \leq i$. We also have $X_{1}\left((p-q) F_{m-1}\right) \equiv\{(\lambda-1) p+(\mu-1) q\}(p-q) F_{m-1} \bmod q^{j}$. If $j \geq 1$, we have $(p-q) F_{m-1} \equiv 0 \bmod q^{j}$ by Lemma 3.8 because $(\lambda-1)+m-3 l+3=$ $3 j-3 l+2 \neq 0$ for every integer $l$ such that $1 \leq l \leq j$. Moreover we have $X_{1}\left((p-q) F_{m-1}\right) \equiv\{(\lambda-1) p+(\mu-1) q\}(p-q) F_{m-1} \bmod (p-q)^{k}$. If $k \geq 1$, we have $(p-q) F_{m-1} \equiv 0 \bmod (p-q)^{k}$ by Lemma 3.6 because $(\lambda-1)+(\mu-1)-m+3 l-3=-3 k+3 l-5 \neq 0$ for every integer $l$ such that $1 \leq l \leq k$. We have $(p-q) F_{m-1} \equiv 0 \bmod p^{i} q^{j}(p-q)^{k}$ by $(6)$. Then there exists an element $c \in K$ such that

$$
(p-q) F_{m-1}=c p^{i} q^{j}(p-q)^{k} .
$$


If we substitute $(31)$ into $(30)$ and notice the relation $(10)_{m}$, we obtain a relation

$$
\{g-2(i-j) t\}(p-q)-(c+2 k t)(p+q)=0 .
$$

Hence we have

$$
\begin{aligned}
g & =2(i-j) t \\
c & =-2 k t
\end{aligned}
$$

From (31) and (33), we find

$$
F_{m-1}=-2 k t p^{i} q^{j}(p-q)^{k-1} .
$$

Step 5 . The polynomial $F_{m-2}$ satisfies the equation $(10)_{m-2}$ :

$(10)_{m-2} \quad X_{1} F_{m-2}=(\lambda p+\mu q) F_{m-2}+g F_{m-1}-X_{0} F_{m-1}-X_{-1} F_{m}$.

If we substitute $(27),(32),(34)$ into $(10)_{m-2}$, we get

$$
\begin{aligned}
X_{1} F_{m-2}= & (\lambda p+\mu q) F_{m-2} \\
& +4 k(k-1) t^{2}(p+q) p^{i} q^{j}(p-q)^{k-2} \\
& +2 i\left(v_{3}-v_{1}\right) p^{i-1} q^{j}(p-q)^{k} \\
& +2 j\left(v_{2}-v_{1}\right) p^{i} q^{j-1}(p-q)^{k} \\
& +2 k\left(1+v_{3}-v_{2}\right) p^{i} q^{j}(p-q)^{k-1} .
\end{aligned}
$$

If $m=1$, we have three cases: (i) $i=1$ and $j=k=0$; (ii) $j=1$ and $i=k=0$; (iii) $k=1$ and $i=j=0$. If the first case holds, we have by (35) $v_{3}-v_{1}=0$. Hence the relation (7) holds in this case. Similarly we have the relation ( 7 ) in the other cases, too. Assume $m \geq 2$. We have

$$
\begin{aligned}
X_{1}\left(p q(p-q)^{2} F_{m-2}\right)= & -(p+q) p q(p-q)^{2} F_{m-2} \\
& +p q(p-q)^{2} X_{1} F_{m-2} .
\end{aligned}
$$

Eliminating $X_{1} F_{m-2}$ from (35) and (36), we have

$$
\begin{aligned}
X_{1}\left(p q(p-q)^{2} F_{m-2}\right)= & \{(\lambda-1) p+(\mu-1) q\} p q(p-q)^{2} \dot{F}_{m-2} \\
& +4 k(k-1) t^{2}(p+q) p^{i+1} q^{j+1}(p-q)^{k} \\
& +2 i\left(v_{3}-v_{1}\right) p^{i} q^{j+1}(p-q)^{k+2} \\
& +2 j\left(v_{2}-v_{1}\right) p^{i+1} q^{j}(p-q)^{k+2} \\
& +2 k\left(1+v_{3}-v_{2}\right) p^{i+1} q^{j+1}(p-q)^{k+1} .
\end{aligned}
$$


We have $X_{1}\left(p q(p-q)^{2} F_{m-2}\right) \equiv\{(\lambda-1) p+(\mu-1) q\} p q(p-q)^{2} F_{m-2} \bmod p^{i}$. If $i \geq 1$, we have $p q(p-q)^{2} F_{m-2} \equiv 0 \bmod p^{i}$ by Lemma 3.7 because $(\mu-1)+$ $(m+2)-3 l+3=3 i-3 l+4 \neq 0$ for every integer $l$ such that $1 \leq l \leq i$. We also have $X_{1}\left(p q(p-q)^{2} F_{m-2}\right) \equiv\{(\lambda-1) p+(\mu-1) q\} p q(p-q)^{2} F_{m-2} \bmod q^{j}$. If $j \geq 1$, we have $p q(p-q)^{2} F_{m-2} \equiv 0 \bmod q^{j}$ by Lemma 3.8 because $(\lambda-1)+(m+2)-3 l+3=3 j-3 l+4 \neq 0$ for every integer $l$ such that $1 \leq l \leq j$. Moreover we have $X_{1}\left(p q(p-q)^{2} F_{m-2}\right) \equiv\{(\lambda-1) p+(\mu-1) q\} p q(p-$ $q)^{2} F_{m-2} \bmod (p-q)^{k}$. If $k \geq 1$, we have $p q(p-q)^{2} F_{m-2} \equiv 0 \bmod (p-q)^{k}$ by Lemma 3.6 because $(\lambda-1)+(\mu-1)-(m+2)+3 l-3=-3 k+3 l-7 \neq 0$ for every integer $l$ such that $1 \leq l \leq k$. We have $p q(p-q)^{2} F_{m-2} \equiv$ $0 \bmod p^{i} q^{j}(p-q)^{k}$ by (6). Then there exists a polynomial $A \in R_{2}$ such that

$$
p q(p-q)^{2} F_{m-2}=A p^{i} q^{j}(p-q)^{k} .
$$

If we substitute (38) into (37) and divide the resulting equation by $p^{i} q^{j}(p-$ $q)^{k}$, then we obtain an equation for $A$ :

$$
\begin{aligned}
L(A)= & 4 k(k-1) t^{2}(p+q) p q \\
& +2 i\left(v_{3}-v_{1}\right) q(p-q)^{2} \\
& +2 j\left(v_{2}-v_{1}\right) p(p-q)^{2} \\
& +2 k\left(1+v_{3}-v_{2}\right) p q(p-q),
\end{aligned}
$$

where we put $L(A)=X_{1} A+(p+q) A$. Three vectors $p q, p(p-q), q(p-q)$ form a basis of the $K$-linear space $R_{2}$, and four vectors $(p+q) p q, p(p-q)^{2}$, $q(p-q)^{2}, p q(p-q)$ form a basis of the $K$-linear space $R_{3}$. Hence, if we consider the formulae

$$
\begin{aligned}
L(p q) & =2(p+q) p q \\
L(p(p-q)) & =p q(p-q)-p(p-q)^{2}, \\
L(q(p-q)) & =p q(p-q)+q(p-q)^{2},
\end{aligned}
$$

then we see that $L$ defines an injective $K$-linear mapping of $R_{2}$ into $R_{3}$. Since $A \in R_{2}$, we can set

$$
A=\xi p q+\eta p(p-q)+\zeta q(p-q)
$$

with $\xi, \eta, \zeta \in K$. If we substitute (43) into (39), then we have

$$
\xi=2 k(k-1) t^{2}
$$




$$
\begin{aligned}
\eta & =-2 j\left(v_{2}-v_{1}\right), \\
\zeta & =2 i\left(v_{3}-v_{1}\right), \\
\eta+\zeta & =2 k\left(1+v_{3}-v_{2}\right) .
\end{aligned}
$$

From (45), (46), (47), we find the expected relation (7). Hence, Proposition 3.5 is proved.

Moreover, let us determine the polynomial $F_{m-2}$, which we will use in the next subsection. Substituting (44), (45), (46) into (43), we get the explicit form of $A$ :

$$
A=2 k(k-1) t^{2} p q-2 j\left(v_{2}-v_{1}\right) p(p-q)+2 i\left(v_{3}-v_{1}\right) q(p-q)
$$

If $i=0, p$ divides $A$. If $j=0, q$ divides $A$. If $k=1, p-q$ divides $A$. If $k=0,(p-q)^{2}$ divides $A$ because of the relation (7). If we substitute (48) into $(38)$ and divide both sides by $p q(p-q)^{2}$, we get

$$
\begin{aligned}
F_{m-2}= & 2 k(k-1) t^{2} p^{i} q^{j}(p-q)^{k-2} \\
& -2 j\left(v_{2}-v_{1}\right) p^{i} q^{j-1}(p-q)^{k-1} \\
& +2 i\left(v_{3}-v_{1}\right) p^{i-1} q^{j}(p-q)^{k-1} .
\end{aligned}
$$

Corollary 3.9. The vector $\mathbf{v}$ in Proposition 3.5 does not belong to the set $\Gamma-W$.

Proof. It is sufficient to show that, for arbitrary non-negative integers $i, j, k$ such that $i+j+k \geq 1$, a complex line in $V$

$$
i\left(v_{1}-v_{3}\right)+j\left(v_{2}-v_{1}\right)+k\left(1+v_{3}-v_{2}\right)=0
$$

does not intersect $\Gamma-W$. Assume the contrary. There exist non-negative integers $i, j, k$ with $i+j+k \geq 1$ and a vector $\mathbf{v}=\left(v_{1}, v_{2}, v_{3}\right) \in \Gamma-W$ such that the relation (50) holds. From (50) we have

$$
i \Re\left(v_{1}-v_{3}\right)+j \Re\left(v_{2}-v_{1}\right)+k \Re\left(1+v_{3}-v_{2}\right)=0
$$

and

$$
i \Im\left(v_{1}-v_{3}\right)+j \Im\left(v_{2}-v_{1}\right)+k \Im\left(v_{3}-v_{2}\right)=0 .
$$

The rest of the proof is divided into three cases:

(i) If the three real parts $\Re\left(v_{1}-v_{3}\right), \Re\left(v_{2}-v_{1}\right)$, and $\Re\left(1+v_{3}-v_{2}\right)$ are not equal to zero, then they are positive because $\mathbf{v} \in \Gamma$. Hence we have 
$i=j=k=0$ by (51). This is a contradiction.

(ii) Assume that one of the real parts is equal to zero and the others are not equal to zero. We assume, for example, $\Re\left(v_{1}-v_{3}\right)=0$ and $\Re\left(v_{2}-\right.$ $\left.v_{1}\right) \Re\left(1+v_{3}-v_{2}\right) \neq 0$ because we can similarly treat the other cases. The two non-zero real parts are positive because $\mathbf{v} \in \Gamma$. We have $j=k=0$ by (51). Since $\mathbf{v} \notin W$, the imaginary part $\Im\left(v_{1}-v_{3}\right)$ is positive. Therefore we have $i=0$ by (52). This is a contradiction.

(iii) Assume that two of the real parts are equal to zero and the other is not equal to zero. Then we can deduce a contradiction by the same argument as above. We omit the detail.

\subsection{Classical solutions}

In the following two lemmas we determine all the non-trivial $X(\mathbf{v})$ invariant principal ideals of $K[p, q]$ for $\mathbf{v} \in \Gamma \cap W$. First we prove the

LEMMA 3.10. (i) Let $\mathbf{v}_{1}$ be a vector in $\Gamma \cap\left\{\mathbf{v} \in V \mid v_{1}=v_{3}\right\}$ and not in D. For every positive integer $i$, a principal ideal $\left(p^{i}\right)$ is $X\left(\mathbf{v}_{1}\right)$ invariant. Conversely, if $I$ is an $X\left(\mathbf{v}_{1}\right)$-invariant principal ideal properly between the zero-ideal and $K[p, q]$, then there exists a positive integer $i$ such that $I=\left(p^{i}\right)$.

(ii) Let $\mathbf{v}_{2}$ be a vector in $\Gamma \cap\left\{\mathbf{v} \in V \mid v_{1}=v_{2}\right\}$ and not in $D$. For every positive integer $j$, a principal ideal $\left(q^{j}\right)$ is $X\left(\mathbf{v}_{2}\right)$-invariant. Conversely, if $I$ is an $X\left(\mathbf{v}_{2}\right)$-invariant principal ideal properly between the zero-ideal and $K[p, q]$, then there exists a positive integer $j$ such that $I=\left(q^{j}\right)$.

(iii) Let $\mathbf{v}_{3}$ be a vector in $\Gamma \cap\left\{\mathbf{v} \in V \mid v_{2}=v_{3}+1\right\}$ and not in $D$. For every positive integer $k$, a principal ideal $\left((p-q-2 t)^{k}\right)$ is $X\left(\mathbf{v}_{3}\right)$ invariant. Conversely, if $I$ is an $X\left(\mathbf{v}_{3}\right)$-invariant principal ideal properly between the zero-ideal and $K[p, q]$, then there exists a positive integer $k$ such that $I=\left((p-q-2 t)^{k}\right)$.

Proof. Since we can similarly prove the remaing assertions, we prove only the assertion (i). The first half is obvious. For the second half, the notation being as in the proof of Proposition 3.5, it is sufficient to show that the $X\left(\mathbf{v}_{1}\right)$-invariant polynomial $F$ is of the form $F=p^{i}$ for some positive integer $i$. We put $v_{1}=\left(v_{1}, v_{2}, v_{3}\right)$. Since $v_{1}=v_{3}$, we have

$$
j\left(v_{2}-v_{1}\right)+k\left(1+v_{3}-v_{2}\right)=0
$$

by (7). Then we have

$$
j \Re\left(v_{2}-v_{1}\right)+k \Re\left(1+v_{3}-v_{2}\right)=0
$$


and

$$
j \Im\left(v_{2}-v_{1}\right)+k \Im\left(v_{3}-v_{2}\right)=0 .
$$

Since $\mathbf{v}_{1}$ is in $\Gamma \cap\left\{\mathbf{v} \in V \mid v_{1}=v_{3}\right\}$ and not in $D$, we have $j=k=0$ by the same argument as in the proof of Corollary 3.9. We have $i=m \geq 1$, $\lambda=-m, \mu=2 m, F_{m}=p^{m}, F_{m-1}=F_{m-2}=0$ by (24), (25), (26), (27), $(34),(49)$. Then the assertion (i) follows immediately from $(10)_{d}$ and the following

Sublemma. Let $d$ be an integer such that $0 \leq d<m$ and let $A$ be a polynomial in $R_{d}$. If $A$ satisfies an equation

$$
X_{1} A=(-m p+2 m q) A,
$$

then $A=0$.

In fact, if $2 m+d \not \equiv 0 \bmod 3$, then we have $2 m+d-3 l+3 \neq 0$ for every integer $l \geq 1$. Hence, by Lemma 3.7, we have $A=0$. If $2 m+d \equiv 0 \bmod 3$, then $k=(2 m+d) / 3$ is a positive integer and we have $2 m+d-3 l+3 \neq 0$ for every integer $l$ such that $1 \leq l \leq k$. It follows from Lemma 3.7 that $A \equiv 0 \bmod p^{k}$. Since $k>d$, we have $A=0$.

Now we prove the

LEMma 3.11. (i) For arbitrary non-negative integers $i, j$ such that $i+$ $j \geq 1$, a principal ideal $\left(p^{i} q^{j}\right)$ is $X(\mathbf{0})$-invariant. Conversely, if $I$ is an $X(\mathbf{0})$-invariant principal ideal properly between the zero-ideal and $K[p, q]$, then there exist non-negative integers $i, j$ such that $i+j \geq 1$ and $I=\left(p^{i} q^{j}\right)$. (ii) For arbitrary non-negative integers $i, k$ such that $i+k \geq 1$, a principal ideal $\left(p^{i}(p-q-2 t)^{k}\right)$ is $X(-1 / 3,2 / 3,-1 / 3)$-invariant. Conversely, if $I$ is an $X(-1 / 3,2 / 3,-1 / 3$-invariant principal ideal properly between the zero-ideal and $K[p, q]$, then there exist non-negative integers $i, k$ such that $i+k \geq 1$ and $I=\left(p^{i}(p-q-2 t)^{k}\right)$.

(iii) For arbitrary non-negative integers $j, k$ such that $j+k \geq 1$, a principal ideal $\left(q^{j}(p-q-2 t)^{k}\right)$ is $X(1 / 3,1 / 3,-2 / 3)$-invariant. Conversely, if $I$ is an $X(1 / 3,1 / 3,-2 / 3)$-invariant principal ideal properly between the zero-ideal and $K[p, q]$, then there exist non-negative integers $j, k$ such that $j+k \geq 1$ and $I=\left(q^{j}(p-q-2 t)^{k}\right)$.

Proof. Since we can similarly prove the remaining assertions, we prove only the assertion (i). The first half is obvious. For the second half, the 
notation being as in the proof of Proposition 3.5, it is sufficient to prove that the $X(\mathbf{0})$-invariant polynomial $F$ is of the form $F=p^{i} q^{j}$ for some non-negative integers $i, j$ such that $i+j \geq 1$. Since $\mathbf{v}=0$, we have $k=0$ by (7). We have $i+j=m \geq 1, \lambda=2 j-i, \mu=2 i-j, F_{m}=p^{i} q^{j}$, $F_{m-1}=F_{m-2}=0$ by (24), (25), (26), (27), (34), (49). Then the assertion (i) follows immediately from $(10)_{d}$ and the following

Sublemma. Let $d$ be an integer such that $0 \leq d<i+j$ and let $A$ be a polynomial in $R_{d}$. If $A$ satisfies an equation

$$
X_{1} A=\{(2 j-i) p+(2 i-j) q\} A
$$

then $A=0$.

In fact, if $2 i-j+d \not \equiv 0 \bmod 3$, then we have $2 i-j+d-3 l+3 \neq 0$ for every integer $l \geq 1$. Therefore, by Lemma 3.7 we have $A=0$. If $2 j-i+d \not \equiv 0 \bmod 3$, then we have $2 j-i+d-3 l+3 \neq 0$ for every integer $l \geq 1$. Therefore, by Lemma 3.8, we have $A=0$. Assume $2 i-j+d \equiv 0$ and $2 j-i+d \equiv 0 \bmod 3$. If we set $i_{1}=(2 i-j+d) / 3$ and $j_{1}=(2 j-i+d) / 3$, then we see that $i_{1}$ and $j_{1}$ are non-negative integers and that $i_{1}+j_{1}>d$. If $i_{1} \geq 1$, we have $A \equiv 0 \bmod p^{i_{1}}$ by Lemma 3.7 because $2 i-j+d-3 l+3 \neq 0$ for every integer $l$ such that $1 \leq l \leq i_{1}$. If $j_{1} \geq 1$, we have $A \equiv 0 \bmod q^{j_{1}}$ by Lemma 3.8 because $2 j-i+d-3 l+3 \neq 0$ for every integer $l$ such that $1 \leq l \leq j_{1}$. Therefore, we have $A \equiv 0 \bmod p^{i_{1}} q^{j_{1}}$, and $A=0$.

\subsection{Proof of Theorem 3.3}

The derivation $X(\mathbf{v})$ for every $\mathbf{v} \in \Gamma-W$ satisfies the condition $(\mathrm{J})$ by Corollary 3.9. Hence we see by Theorem 1.1 that every transcendental solution $(p, q)$ of $S_{\mathrm{IV}}(\mathbf{v})$ for all $\mathbf{v} \in \Gamma-W$ is non-classical.

On the other hand, it follows that all the transcendental classical solutions of $S_{\mathrm{IV}}(\mathbf{v})$ for $\mathbf{v} \in \Gamma \cap W$ are determined by the principal prime ideals $(p),(q),(p-q-2 t)$, and that the other transcendental solutions of $S_{\mathrm{IV}}(\mathbf{v})$ for $\mathbf{v} \in \Gamma \cap W$ are not classical. Thus we complete the proof of Theorem 3.3.

\subsection{The Newton polygon of the invariant polynomial $F$}

By definition, the Newton polygon of the derivation $X(\mathbf{v})$ is represented by the following picture: 


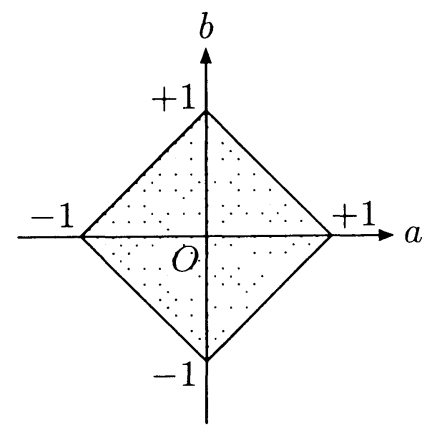

The grading introduced in the proof of Proposition 3.5 comes from the edge joining the vertices $(0,1)$ and $(1,0)$.

On the other hand, by the same argument as in Subsection 2.5, we find the following figure of the the Newton polygon of the invariant polynomial $F$ :

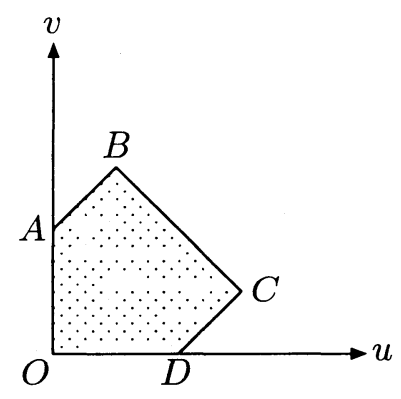

Here an integral point $(u, v)$ in $\mathbf{R}^{2}$ represents a monomial $\gamma p^{u} q^{v}(\gamma \in$ $K)$. In the figure the Cartesian coordinates of the vertices $O, A, B, C, D$ are $(0,0),(0, j+k-i),(i, j+k),(i+k, j),(i+k-j, 0)$. The edge $B C$ represents the polynomial $F_{m}=p^{i} q^{j}(p-q)^{k}$, the edge $A B$ a polynomial $(-1)^{k}(p q+$ $\left.2 v_{1}-2 v_{3}\right)^{i} q^{j+k-i}$, the edge $C D$ a polynomial $\left(p q+2 v_{1}-2 v_{2}\right)^{j} p^{i+k-j}$. Notice that the Newton polygon of $F$ can be degenerate similarly to the case in the second Painlevé equation (see Lemmas 3.10 and 3.11).

\section{REFERENCES}

[1] N. Bourbaki, Groupes et algèbres de Lie, Chapitres 4, 5 et 6, Hermann, Paris, 1968.

[2] B. Gambier, Sur les équations différentielles du second ordre et du premier degré dont l'intégrale générale est à points critiques fixes, Acta Math., 33 (1909), 1-55.

[3] V. I. Gromak, One-parameter systems of solutions of Painlevé's equations, Diff. Eq., 14 (1978), 1510-1513. 
[4] V. I. Gromak, Solutions of the second Painlevé equation, Diff. Eq., 18 (1982), $537-545$.

[5] V. I. Gromak, Theory of the fourth Painlevé equation, Diff. Eq., 23 (1987), 506-513.

[6] V. I. Gromak, Transformations of Painlevé equations, Dokl. Akad. Nauk. BSSR., 32 (1988), 395-398, (in Russian).

[7] V. I. Gromak, Transformations of Painlevé equatıons $\left(P_{4}\right)-\left(P_{6}\right)$, Dokl. Akad. Nauk. BSSR., 33 (1989), 17-20, (in Russian).

[8] V. I. Gromak, One-parameter families of solutions of the second Painlevé equation, Dokl. Akad. Nauk. BSSR., 33 (1989), 209-211, (in Russian).

[9] V. I. Gromak and N. A. Lukashevich, Special classes of solutions of Painlevé's equations, Diff. Eq., 18 (1982), 317-326.

[10] V. I. Gromak and V. V. Tsegel'nik, Solutions of Painlevé's fourth equation and their applications, Diff. Eq., 27 (1991), 910-918.

[11] E. L. Ince, Ordinary differential equations, Dover.

[12] E. R. Kolchin, Differential Algebra and Algebraic groups, Academic Press, 1973.

[13] N. A. Lukashevich, Theory of the fourth Painlevé equation, Diff. Eq., 3 (1967), 395-399.

[14] N. A. Lukashevich, The second Painlevé equation, Diff. Eq., 7 (1971), 853-854.

[15] Y. Murata, Classical solutions of the third Painlevé equation, Nagoya Math. J., 139 (1995), 37-65.

[16] Y. Murata, Rational solutions of the second and fourth Painlevé equations, Funk. Ekvac., 28 (1985), 1-32.

[17] K. Nishioka, A note on the transcendency of Painlevé's first transcendent, Nagoya Math. J., 109 (1988), 63-67.

[18] M. Noumi, Private communications (1986 and 1987).

[19] M. Noumi and K. Okamoto, Irreducibility of the second and the fourth Painlevé equations, Funk. Ekvac., 40 (1997), 139-163.

[20] K. Okamoto, Studies on the Painlevé equations III, second and fourth Painlevé equations, $P_{\mathrm{II}}$ and $P_{\mathrm{IV}}$, Math. Ann., 275 (1986), 221-255.

[21] K. Okamoto, Private communication (1987).

[22] P. Painlevé, Mémoire sur les équations différentielles dont l'intégrale générale est uniforme, Bull. Soc. Math. France, 28 (1900), 201-261.

[23] P. Painlevé, Sur les équations différentielles du second ordre et d'ordre supérieur dont l'intégrale générale est uniforme, Acta Math., 25 (1900), 1-85.

[24] P. Painlevé, C. R. Acad. Sci. Paris, 143 (1906), 1111-1117.

[25] P. Painlevé, Leçons de Stockholm, Euvres de P. Painlevé I, Editions du C. N. R. S., Paris, 1972.

[26] H. Umemura, Birational automorphism groups and differential equations, Nagoya Math. J., 119 (1990), 1-80.

[27] H. Umemura, On the irreducibility of the first differential equation of Painlevé, Algebraic Geometry and Commutative Algebra in Honor of Masayoshi NAGATA (1987), Kinokuniya, Tokyo, 771-789.

[28] H. Umemura, Second proof of the irreducibility of first differential equation of Painlevé, Nagoya Math. J., 117 (1990), 125-171. 
[29] H. Umemura, Differential Galois theory of infinite dimension, Nagoya Math. J., 144 (1996), 59-135.

[30] H. Umemura and H. Watanabe, Solutions of the second and fourth Painleve equations II, in preparation.

[31] Vorob'ev, A. P., On rational solutions of the second Painlevé equation, Diff. Eq., 1 (1965), 58-59.

[32̇] H. Watanabe, Solutions of the fifth Painlevé equation I, Hokkaido Math. J., 24 (1995), 231-267..

[33] A. I. Yablonskii, On rational solutions of the second Painlevé equation, Vesti A. N. BSSR. Ser. Fiz-Tekh. Nauk., 3 (1959), 30-35, (in Russian).

Hiroshi Umemura

Graduate School of Polymathematics

Nagoya University

Nagoya 464-01, Japan

umemura@math.nagoya-u.ac.jp

Humihiko Watanabe

Graduate School of Mathematics

Kyushu University

Fukuoka 810, Japan 